\title{
Local Model Checking of Weighted CTL with Upper-Bound Constraints
}

\author{
Jonas Finnemann Jensen, Kim Guldstrand Larsen, \\ Jiří Srba, and Lars Kaerlund Oestergaard \\ Department of Computer Science, Aalborg University \\ Selma Lagerlöfs Vej 300, 9220 Aalborg, Denmark \\ jopsen@gmail.com, \{kgl,srba\}@cs.aau.dk, larsko@gmail.com
}

\begin{abstract}
We present a symbolic extension of dependency graphs by Liu and Smolka in order to model-check weighted Kripke structures against the logic CTL with upper-bound weight constraints. Our extension introduces a new type of edges into dependency graphs and lifts the computation of fixed-points from boolean domain to nonnegative integers in order to cope with the weights. We present both global and local algorithms for the fixed-point computation on symbolic dependency graphs and argue for the advantages of our approach compared to the direct encoding of the model checking problem into dependency graphs. We implement all algorithms in a publicly available tool prototype and evaluate them on several experiments. The principal conclusion is that our local algorithm is the most efficient one with an order of magnitude improvement for model checking problems with a high number of "witnesses".
\end{abstract}

\section{Introduction}

Model-driven development is finding its way into industrial practice within the area of embedded systems. Here a key challenge is how to handle the growing complexity of systems, while meeting requirements on correctness, predictability, performance and not least time- and cost-to-market. In this respect modeldriven development is seen as a valuable and promising approach, as it allows early design-space exploration and verification and may be used as the basis for systematic and unambiguous testing of a final product. However, for embedded systems, verification should not only address functional properties but also a number of non-functional properties related to timing and resource constraints.

Within the area of model checking a number of state-machine based modeling formalisms has emerged, allowing for such quantitative aspects to be expressed. In particular, timed automata (TA) [1], and the extensions to weighted timed automata (WTA) $[6,2]$ are popular and tool-supported formalisms that allow for such constraints to be modeled.

Interesting behavioural properties of TAs and WTAs may be expressed in natural weight-extended versions of classical temporal logics such as CTL for 
branching-time and LTL for linear-time. Just as TCTL and MTL provide extensions of CTL and LTL with time-constrained modalities, WCTL and WMTL are extensions with weight-constrained modalities interpreted with respect to WTAs. Unfortunately, the addition of weight now turns out to come with a price: whereas the model-checking problems for TAs with respect to TCTL and MTL are decidable, it has been shown that model-checking WTAs with respect to WCTL is undecidable [9].

In this paper we reconsider this model checking problem in the setting of untimed models, i.e. essentially weighted Kripke structures, and negation-free WCTL formula with only upper bound constraints on weights. As main contributions, we show that in this setting the model-checking problem is in PTIME, and we provide an efficient symbolic, local (on-the-fly) model checking algorithm.

Our results are based on a novel symbolic extension of the dependency graph framework of Liu and Smolka [16] where they encode boolean equation systems and offer global and local algorithms for computing minimal and maximal fixed points in linear time. Whereas a direct encoding of our model checking problem into dependency graphs leads to a pseudo-polynomial algorithm ${ }^{1}$, the novel symbolic dependency graphs allow for a polynomial encoding and a polynomial time fixed-point computation. Most importantly, the symbolic dependency graph encoding enables us to perform a symbolic local fixed-point evaluation. Experiments with the various approaches (direct versus symbolic encoding, global versus local algorithm) have been conducted on a large number of cases, demonstrating that the combined symbolic and local approach is the most efficient one. For model-checking problems with affirmative outcome, this combination is often one order or magnitude faster than the other approaches.

\section{Related Work}

Laroussinie, Markey and Oreiby [14] consider the problem of model checking durational concurrent game structures with respect to timed ATL properties, offering a PTIME result in the case of non-punctual constraints in the formula. Restricting the game structures to a single player gives a setting similar to ours, as timed ATL is essentially WCTL. However, in contrast to [14], we do allow transitions with zero weight in the model, making a fixed-point computation necessary. As a result, the corresponding CTL model checking (with no weight constraints) is a special instance of our approach, which is not the case for [14]. Most importantly, the work in [14] does not provide any local algorithm, which our experiments show is crucial for the performance. No implementation is provided in [14].

Buchholz and Kemper [10] propose a valued computation tree logic (CTL\$) interpreted over a general set of weighted automata that includes CTL in the logic as a special case over the boolean semiring. For model checking CTL $\$$ formulae they describe a matrix-based algorithm. Their logic is more expressive than the one proposed here, since they support negation and all the comparison

\footnotetext{
${ }^{1}$ Exponential in the encoding of the weights in the model and the formula.
} 
operators. In addition, they permit nested CTL formulae and can operate on $\max /$ plus semirings in $O(\min (\log (t) \cdot m m, t \cdot n z))$ time, where $t$ is the number of vector matrix products, $m m$ is the complexity of multiplying two matrices of order $n$ and $n z$ is the number of non-zero elements in special matrix used for checking "until" formulae up to some bound $t$. However, they do not provide any on-the-fly technique for verification.

Another related work [8] shows that the model-checking problem with respect to WCTL is PSPACE-complete for one-clock WTAs and for TCTL (the only cost variable is the time elapsed).

Several approaches to on-the-fly/local algorithms for model checking the modal mu-calculus have been proposed. Andersen [3] describes a local algorithm for model checking the modal mu-calculus for alternation depth one running in $O(n \cdot \log (n))$ (where $n$ is the product of the size of the assertion and the labeled transition system). Liu and Smolka[16] improve on the complexity of this approach with a local algorithm running in $O(n)$ (where $n$ is the size of the input graph) for evaluating alternation-free fixed points. This is also the algorithm that we apply for WCTL model checking and the one we extend for symbolic dependency graphs. Cassez et. al. [11] present another symbolic extension of the algorithm by Liu and Smolka; a zone-based forward, local algorithm for solving timed reachability games. Later Liu, Ramakrishnan and Smolka [15] also introduce a local algorithm for the evaluation of alternating fixed points with the complexity $O\left(n+\left(\frac{n+a d}{a d}\right)^{a d}\right)$, where $a d$ is the alternation depth of the graph. We do not consider the evaluation of alternating fixed points in the weighted setting and this is left for the future work.

Outline. Weighted Kripke structures and weighted CTL (WCTL) are presented in Section 2. Section 3 then introduces dependency graphs. Model checking WCTL with this framework is discussed in Section 4. In Section 5 we propose symbolic dependency graphs and demonstrate how they can be used for WCTL model checking in Section 6. Experimental results are presented in Section 7 and Section 8 concludes the paper.

\section{Basic Definitions}

Let $\mathbb{N}_{0}$ be the set of nonnegative integers. A Weighted Kripke Structure (WKS) is a quadruple $\mathcal{K}=(S, \mathcal{A P}, L, \rightarrow)$, where $S$ is a finite set of states, $\mathcal{A P}$ is a finite set of atomic propositions, $L: S \rightarrow \mathcal{P}(\mathcal{A P})$ is a mapping from states to sets of atomic propositions, and $\rightarrow \subseteq S \times \mathbb{N}_{0} \times S$ is a transition relation.

Instead of $\left(s, w, s^{\prime}\right) \in \rightarrow$, meaning that from the state $s$, under the weight $w$, we can move to the state $s^{\prime}$, we often write $s \stackrel{w}{\rightarrow} s^{\prime}$. A WKS is nonblocking if for every $s \in S$ there is an $s^{\prime}$ such that $s \stackrel{w}{\rightarrow} s^{\prime}$ for some weight $w$. From now on we consider only nonblocking $\mathrm{WKS}^{2}$.

\footnotetext{
${ }^{2}$ A blocking WKS can be turned into a nonblocking one by introducing a new state with no atomic propositions, zero-weight self-loop and with zero-weight transitions from all blocking states into this newly introduced state.
} 
A run in an WKS $\mathcal{K}=(S, \mathcal{A P}, L, \rightarrow)$ is an infinite computation

$$
\sigma=s_{0} \stackrel{w_{0}}{\rightarrow} s_{1} \stackrel{w_{1}}{\rightarrow} s_{2} \stackrel{w_{2}}{\rightarrow} s_{3} \ldots
$$

where $s_{i} \in S$ and $\left(s_{i}, w_{i}, s_{i+1}\right) \in \rightarrow$ for all $i \geq 0$. Given a position $p \in \mathbb{N}_{0}$ in the run $\sigma$, let $\sigma(p)=s_{p}$. The accumulated weight of $\sigma$ at position $p \in \mathbb{N}_{0}$ is then defined as $W_{\sigma}(p)=\Sigma_{i=0}^{p-1} w_{i}$.

We can now define negation-free Weighted Computation Tree Logic (WCTL) with weight upper-bounds. The set of WCTL formulae over the set of atomic propositions $\mathcal{A P}$ is given by the abstract syntax

$$
\begin{aligned}
& \varphi::=\text { true } \mid \text { false }|a| \varphi_{1} \wedge \varphi_{2}\left|\varphi_{1} \vee \varphi_{2}\right| \\
& \qquad E X_{\leq k} \varphi\left|A X_{\leq k} \varphi\right| E \varphi_{1} U_{\leq k} \varphi_{2} \mid A \varphi_{1} U_{\leq k} \varphi_{2}
\end{aligned}
$$

where $k \in \mathbb{N}_{0} \cup\{\infty\}$ and $a \in \mathcal{A P}$. We assume that the $\infty$ element added to $\mathbb{N}_{0}$ is larger than any other natural number and that $\infty+k=\infty-k=\infty$ for all $k \in \mathbb{N}_{0}$. We now inductively define the satisfaction triple $s=\varphi$, meaning that a state $s$ in an implicitly given WKS satisfies a formula $\varphi$.

$$
\begin{array}{ll}
s \models \text { true } & \text { if } a \in L(s) \\
s \models a & \text { if } s \models \varphi_{1} \text { and } s \models \varphi_{2} \\
s \models \varphi_{1} \wedge \varphi_{2} & \text { if } s \models \varphi_{1} \text { or } s \models \varphi_{2} \\
s \models \varphi_{1} \vee \varphi_{2} & \text { if there exists a run } \sigma \text { starting from } s \text { and a position } p \geq 0 \\
s \models E \varphi_{1} U_{\leq k} \varphi_{2} & \text { s.t. } \sigma(p) \models \varphi_{2}, W_{\sigma}(p) \leq k \text { and } \sigma\left(p^{\prime}\right) \models \varphi_{1} \text { for all } p^{\prime}<p \\
& \text { if for any run } \sigma \text { starting from } s, \text { there is a position } p \geq 0 \\
s \models A \varphi_{1} U_{\leq k} \varphi_{2} & \text { s.t. } \sigma(p) \models \varphi_{2}, W_{\sigma}(p) \leq k \text { and } \sigma\left(p^{\prime}\right) \models \varphi_{1} \text { for all } p^{\prime}<p \\
s \models E X_{\leq k} \varphi & \text { if } \exists s^{\prime} \text { s.t. } s \stackrel{w}{\rightarrow} s^{\prime}, s^{\prime} \models \varphi \text { and } w \leq k \\
s \models A X_{\leq k} \varphi & \text { if } \forall s^{\prime} \text { s.t. } s \stackrel{w}{\rightarrow} s^{\prime} \text { where } w \leq k \text { it holds that } s^{\prime} \models \varphi
\end{array}
$$

\section{Dependency Graph}

In this section we present the dependency graph framework and a local algorithm for minimal fixed-point computation as originally introduced by Liu and Smolka [16]. This framework can be applied to model checking of the alternationfree modal mu-calculus, including the CTL logic. Later, in Section 4, we demonstrate how to extend the framework from CTL to WCTL.

Definition 1 (Dependency Graph). A dependency graph is a pair $G=$ $(V, E)$ where $V$ is a finite set of configurations, and $E \subseteq V \times \mathcal{P}(V)$ is a finite set of hyper-edges.

Let $G=(V, E)$ be a dependency graph. For a hyper-edge $e=(v, T)$, we call $v$ the source configuration and $T$ the target (configuration) set of $e$. For a configuration $v$, the set of its successors is given by $\operatorname{succ}(v)=\{(v, T) \in E\}$. 


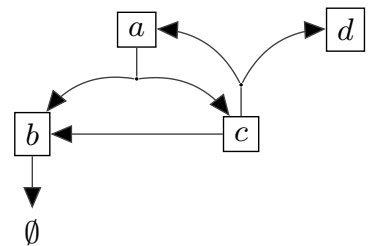

$$
\begin{aligned}
& a=b \wedge c \\
& c=b \vee(a \wedge d) \\
& b=\text { true }
\end{aligned}
$$

\begin{tabular}{r|cccc} 
& $a$ & $b$ & $c$ & $d$ \\
\hline$A_{0}$ & 0 & 0 & 0 & 0 \\
$F\left(A_{0}\right)$ & 0 & 1 & 0 & 0 \\
$F^{2}\left(A_{0}\right)$ & 0 & 1 & 1 & 0 \\
$F^{3}\left(A_{0}\right)$ & 1 & 1 & 1 & 0 \\
$F^{4}\left(A_{0}\right)$ & 1 & 1 & 1 & 0
\end{tabular}

Fig. 1. A dependency graph, function $F$, and four iterations of the global algorithm

An assignment $A: V \rightarrow\{0,1\}$ is a function that assigns boolean values to configurations of $G$. A pre fixed-point assignment of $G$ is an assignment $A$ where, for every configuration $v \in V$, holds that if $(v, T) \in E$ and $A(u)=1$ for all $u \in T$ then also $A(v)=1$.

By taking the standard component-wise ordering $\sqsubseteq$ on assignments, where $A \sqsubseteq A^{\prime}$ if and only if $A(v) \leq A^{\prime}(v)$ for all $v \in V$ (assuming that $0<1$ ), we get by Knaster-Tarski fixed-point theorem that there exists a unique minimum pre fixed-point assignment, denoted by $A_{\min }$.

The minimum pre fixed-point assignment $A_{\min }$ of $G$ can be computed by repeated applications of the monotonic function $F$ from assignments to assignments, starting from $A_{0}$ where $A_{0}(v)=0$ for all $v \in V$, and where

$$
F(A)(v)=\bigvee_{(v, T) \in E}\left(\bigwedge_{u \in T} A(u)\right)
$$

for all $v \in V$. We are guaranteed to reach a fixed point after a finite number of applications of $F$ due to the finiteness of the complete lattice of assignments ordered by $\sqsubseteq$. Hence there exists an $m \in \mathbb{N}_{0}$ such that $F^{m}\left(A_{0}\right)=F^{m+1}\left(A_{0}\right)$, in which case we have $F^{m}\left(A_{0}\right)=A_{\min }$. We will refer to this algorithm as the global one.

Example 1. Figure 1 shows a dependency graph, its corresponding function $F$ given as a boolean equation system, and four iterations of the global algorithm (sufficient to compute the minimum pre fixed-point assignment). Configurations in the dependency graph are illustrated as labeled squares and hyper-edges are drawn as a span of lines to every configuration in the respective target set.

In model checking we are often only interested in the minimum pre-fixed point assignment $A_{\min }(v)$ for a specific configuration $v \in V$. For this purpose, Liu and Smolka [16] suggest a local algorithm presented with minor modifications ${ }^{3}$ in Algorithm 1. The algorithm maintains three data-structures throughout its execution: an assignment $A$, a dependency set $D$ for every configuration and a set of hyper-edges $W$. The dependency set $D(v)$ for a configuration $v$ maintains

\footnotetext{
${ }^{3}$ At line 12 we added the current hyper-edge $e$ to the dependency set $D(u)$ of the successor configuration $u$, i.e. $D(u)=\{e\}$. The original algorithm sets the dependency set to empty here, leading to an incorrect propagation.
} 


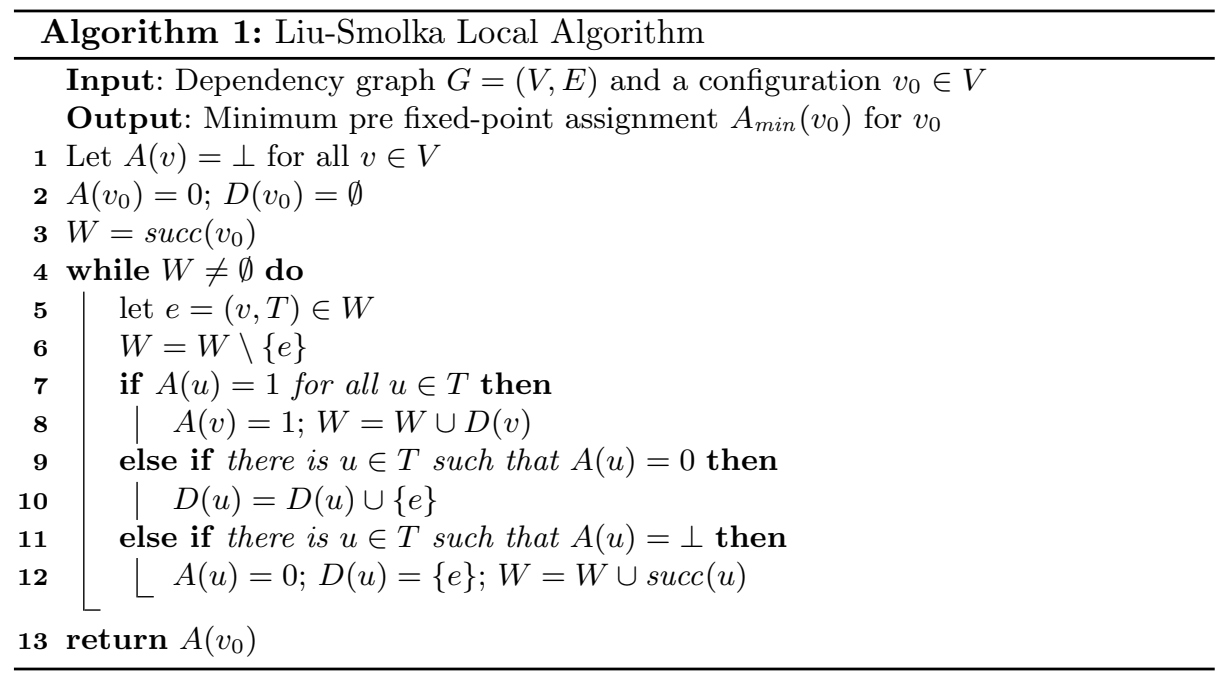

a list of hyper-edges that were processed under the assumption that $A(v)=0$. Whenever the value of $A(v)$ changes to 1, the hyper-edges from $D(v)$ must be reprocessed in order to propagate this change to the respective sources of the hyper-edges.

Theorem 1 (Correctness of Local Algorithm [16]). Given a dependency graph $G=(V, E)$ and a configuration $v_{0} \in V$, Algorithm 1 computes the minimum pre-fixed point assignment $A_{\min }\left(v_{0}\right)$ for the configuration $v_{0}$.

As argued in [16], both the local and global model checking algorithms run in linear time.

\section{Model Checking with Dependency Graphs}

In this section we suggest a reduction from the model checking problem of WCTL (on WKS) to the computation of minimum pre fixed-point assignment on a dependency graph.

Given a WKS $\mathcal{K}$, a state $s$ of $\mathcal{K}$, and a WCTL formula $\varphi$, we construct a dependency graph where every configuration is a pair of a state and a formula. Starting from the initial pair $\langle s, \varphi\rangle$, the dependency graph is constructed according to the rules given in Figure 2.

Theorem 2 (Encoding Correctness). Let $\mathcal{K}=(S, \mathcal{A P}, L, \rightarrow)$ be a WKS, $s \in S$ a state, and $\varphi$ a WCTL formula. Let $G$ be the constructed dependency graph rooted with $\langle s, \varphi\rangle$. Then $s \models \varphi$ if and only if $A_{\min }(\langle s, \varphi\rangle)=1$.

Proof. By structural induction on the formula $\varphi$. Details are given in the appendix. 


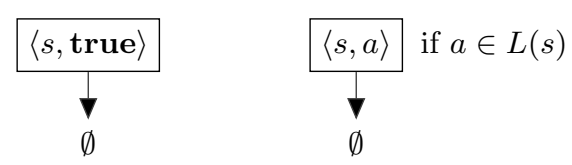

(a) True (b) Proposition

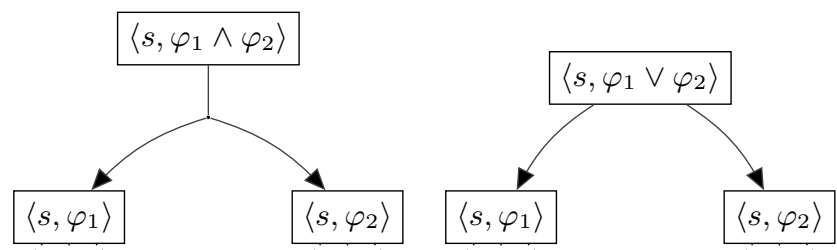

(c) Conjunction

(d) Disjunction

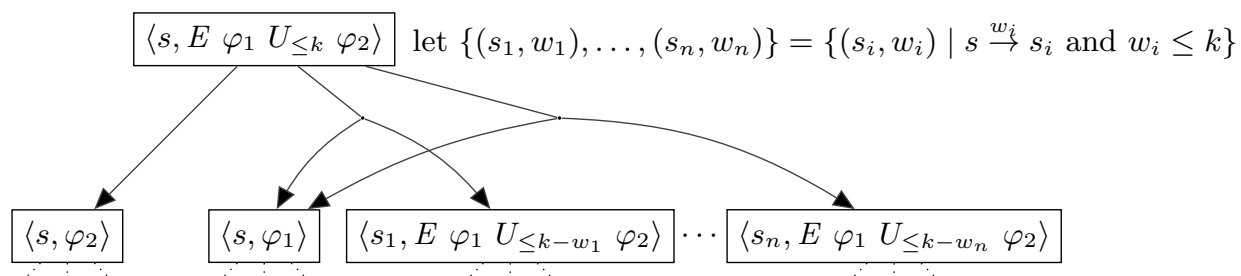

(e) Existential Until

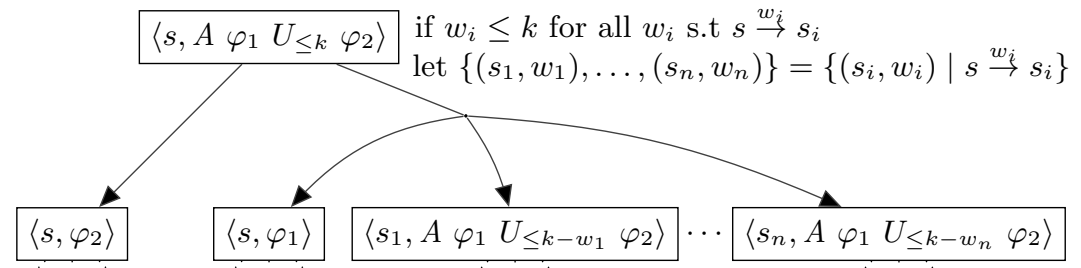

(f) Universal Until

let $\left\{s_{1}, s_{2}, \ldots, s_{n}\right\}=\left\{s_{i} \mid s \stackrel{w_{i}}{\rightarrow} s_{i}, w_{i} \leq k\right\}$

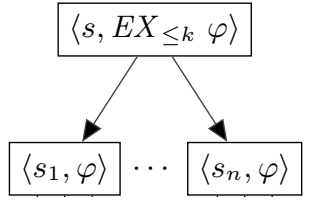

(g) Existential Next

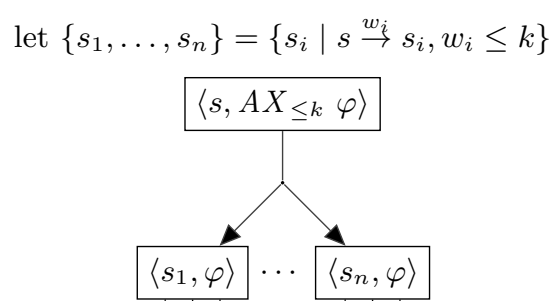

(h) Universal Next

Fig. 2. Dependency graph encoding of state-formula pairs. 


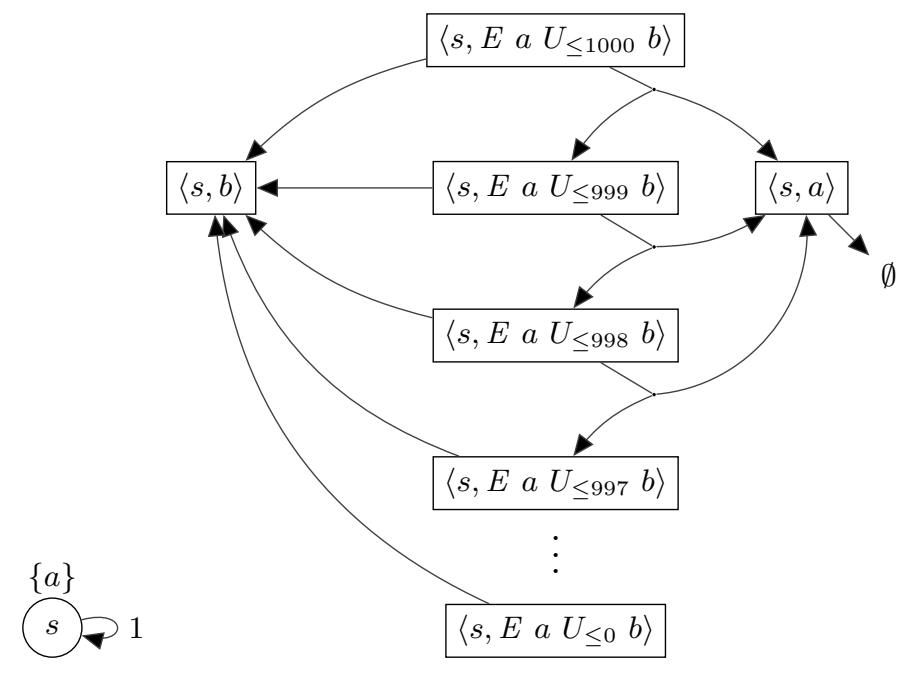

Fig. 3. A WKS and its dependency graph for the formula $E$ a $U_{\leq 1000} b$

Clearly, to profit from the local algorithm by Liu and Smolka [16] presented in the previous section, we construct the dependency graph on-the-fly whenever successor configurations are requested by the algorithm. Such an exploration gives us often more efficient local model checking algorithm compared to the global one (see Section 7).

However, the drawback of this approach is that we may need to construct exponentially large dependency graphs. This is demonstrated in Figure 3 where a single-state WKS on the left gives rise to a large dependency graph on the right where its size depends on the bound in the formula. Hence this method gives us only a pseudo-polynomial algorithm for model checking WCTL.

\section{Symbolic Dependency Graphs}

We have seen in previous section that the use of dependency graphs for WCTL model checking suffers from the exponential explosion as the graph grows in proportion to the bounds in the given formula (due to the unfolding of the until operators). We can, however, observe that the validity of $s \models E a U_{\leq k} b$ implies $s \models E a U_{\leq k+1} b$. In what follows we suggest a novel extension of dependency graphs, called symbolic dependency graphs, that use the implication above in order to reduce the size of the constructed graphs. Then in Section 6 we shall use symbolic dependency graphs for efficient (polynomial time) model checking of WCTL.

Definition 2 (Symbolic Dependency Graph). A symbolic dependency graph (SDG) is a triple $G=(V, H, C)$, where $V$ is a finite set of configurations, $H \subseteq V \times \mathcal{P}\left(\mathbb{N}_{0} \times V\right)$ is a finite set of hyper-edges, and $C \subseteq V \times \mathbb{N}_{0} \times V$ is a finite set of cover-edges. 


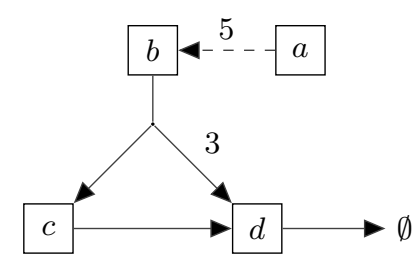

(a) A symbolic dependency graph

\begin{tabular}{r|cccc}
$i$ & $a$ & $b$ & $c$ & $d$ \\
\hline$A_{0}$ & $\infty$ & $\infty$ & $\infty$ & $\infty$ \\
$F\left(A_{0}\right)$ & $\infty$ & $\infty$ & $\infty$ & 0 \\
$F^{2}\left(A_{0}\right)$ & $\infty$ & $\infty$ & 0 & 0 \\
$F^{3}\left(A_{0}\right)$ & $\infty$ & 3 & 0 & 0 \\
$F^{4}\left(A_{0}\right)$ & 0 & 3 & 0 & 0 \\
$F^{5}\left(A_{0}\right)$ & 0 & 3 & 0 & 0
\end{tabular}

(b) Minimum pre fixed-point computation

Fig. 4. Computation of minimum pre fixed-point assignment of a SDG

The difference from dependency graphs explained earlier is that for each hyper-edge of a SDG a weight is added to all of its target configurations and a new type of edge called a cover-edge is introduced. Let $G=(V, H, C)$ be a symbolic dependency graph. The size of $G$ is $|G|=|V|+|H|+|C|$ where $|V|$, $|H|$ and $|C|$ is the size the of these components in a binary representation (note that the size of a hyper-edge depends on the number of nodes it connects to). For a hyper-edge $e=(v, T) \in H$ we call $v$ the source configuration and $T$ the target set of $e$. We also say that $(w, u) \in T$ is a hyper-edge branch with weight $w$ pointing to the target configuration $u$. The successor set $\operatorname{succ}(v)=\{(v, T) \in$ $H\} \cup\{(v, k, u) \in C\}$ is the set of hyper-edges and cover-edges with $v$ as the source configuration.

Figure 4(a) shows an example of a SDG. Hyper-edges are denoted by solid lines and hyper-edge branches have weight 0 unless they are annotated with another weight. Cover-edges are drawn as dashed lines annotated with a covercondition. We shall now describe a global algorithm for the computation of the minimum pre fixed-point. The main difference is that symbolic dependency graphs operate over the complete lattice $\mathbb{N}_{0} \cup\{\infty\}$, contrary to standard dependency graphs that use only boolean values.

An assignment $A: V \rightarrow \mathbb{N}_{0} \cup\{\infty\}$ in an SDG $G=(V, H, C)$ is a mapping from configurations to values. We denote the set of all assignments by Assign. A pre fixed-point assignment is an assignment $A \in A$ Asign such that $A=F(A)$ where $F:$ Assign $\rightarrow$ Assign is defined as

$F(A)(v)= \begin{cases}0 & \text { if } \exists\left(v, k, v^{\prime}\right) \in C \text { s.t. } A\left(v^{\prime}\right) \leq k<\infty, \text { or } A\left(v^{\prime}\right)<k=\infty \\ \min _{(v, T) \in H}\left(\max \left\{w+A\left(v^{\prime}\right) \mid\left(w, v^{\prime}\right) \in T\right\}\right) \quad \text { otherwise. }\end{cases}$

If we consider the partial order $\sqsubseteq$ over assignments of a symbolic dependency graph $G$ such that $A \sqsubseteq A^{\prime}$ if and only if $A(v) \geq A^{\prime}(v)$ for all $v \in V$, then the function $F$ is clearly monotonic on the complete lattice of all assignments ordered by $\sqsubseteq$. It follows by Knaster-Tarski fixed-point theorem that there exists a unique minimum pre fixed-point assignment of $G$, denoted $A_{\text {min }}$. 
Notice that we write $A \sqsubseteq A^{\prime}$ if for all configurations $v$ we have $A(v) \geq A^{\prime}(v)$ in the opposite order. Hence, $A_{0}(v)=\infty$ for all $v \in V$ is the smallest element in the lattice.

As the lattice is finite and there are no infinite decreasing sequences of weights (nonnegative integers), the minimum pre fixed-point assignment $A_{\min }$ of $G$ can be computed by a finite number of applications of the function $F$ on the smallest assignment $A_{0}$, where all configurations have the initial value $\infty$. So there exists an $m \in \mathbb{N}_{0}$ such that $F^{m}\left(A_{0}\right)=F^{m+1}\left(A_{0}\right)$, implying that $F^{m}\left(A_{0}\right)=A_{\text {min }}$ is the minimum pre fixed-point assignment of $G$. Figure 4(b) shows a computation of the minimum pre fixed-point assignment on our example.

The next theorem demonstrates that fixed-point computation via the global algorithm (repeated applications of the function $F$ ) on symbolic dependency graphs still runs in polynomial time (proof is in the appendix).

Theorem 3. The computation of the minimum post fixed-point assignment for an $S D G G=(V, H, C)$ by repeated application of the function $F$ takes time $O(|V| \cdot|C| \cdot(|H|+|C|))$.

We now propose a local algorithm for minimum pre fixed-point computation on symbolic dependency graphs, motivated by the fact that in model checking we are often interested in the value for a single given configuration only, hence we might be able (depending on the formula we want to verify) to explore only a part of the reachable state space.

Given a symbolic dependency graph $G=(V, H, C)$, Algorithm 2 computes the minimum pre fixed-point assignment $A_{\min }\left(v_{0}\right)$ of a configuration $v_{0} \in V$. The algorithm is an adaptation of Algorithm 1 . We use the same data-structures as in Algorithm 1. However, the assignment $A(v)$ for each configuration $v$ now ranges over $\mathbb{N}_{0} \cup\{\perp, \infty\}$ where $\perp$ once again indicates that the value is unknown at the moment.

Table 1 lists the values of the assignment $A$, the set $W$ (implemented as queue) and the dependency set $D$ during the execution of Algorithm 2 on the SDG Figure 4(a). Each row displays the values before the $i$ 'th iteration of the while-loop. The value of the dependency set $D(a)$ for $a$ is not shown in the table because it remains empty.

In order to prove the correctness of Algorithm 2, we extend the loop invariant for the local algorithm on dependency graphs [16] with weights. The proof of the invariant is given in the appendix.

Lemma 1. The while-loop in Algorithm 2 satisfies the following loop-invariants (for all configurations $v \in V$ ):

1) If $A(v) \neq \perp$ then $A(v) \geq A_{\min }(v)$.

2) If $A(v) \neq \perp$ and $e=(v, T) \in H$, then either

a) $e \in W$,

b) $e \in D(u)$ and $A(v) \leq x$ for some $(w, u) \in T$ s.t. $x=A(u)+w$, where $x \geq A\left(u^{\prime}\right)+w^{\prime}$ for all $\left(w^{\prime}, u^{\prime}\right) \in T$, or

c) $A(v)=0$. 


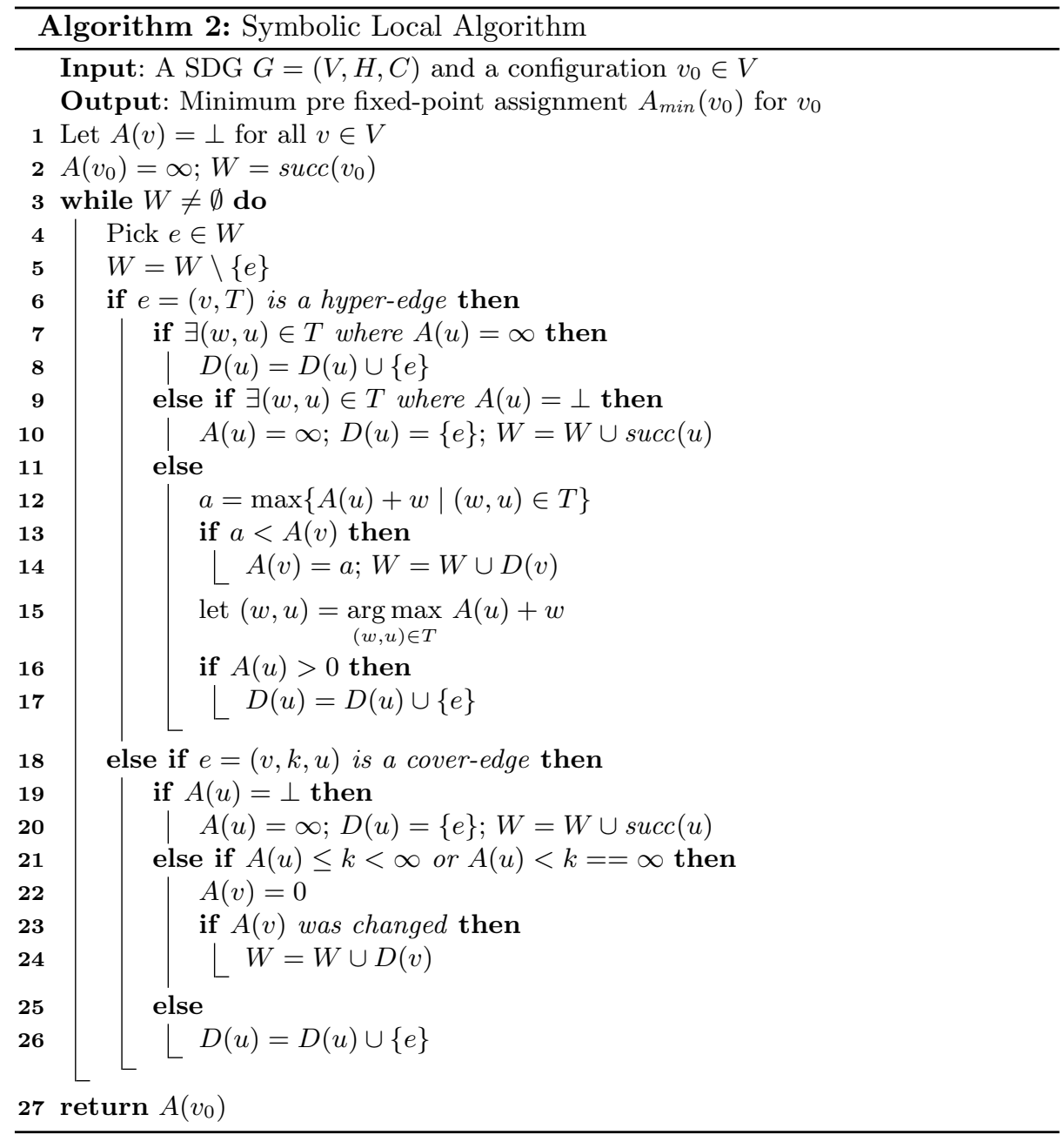

3) If $A(v) \neq \perp$ and $e=(v, k, u) \in C$, then either

a) $e \in W$,

b) $e \in D(u)$ and $A(u)>k$, or

c) $A(v)=0$.

These loop-invariants allow us to conclude the correctness of the local algorithm (details are in the appendix).

Theorem 4. Algorithm 2 terminates and computes an assignment $A$ such that $A(v) \neq \perp$ implies $A(v)=A_{\text {min }}(v)$ for all $v \in V$. In particular, the returned value $A\left(v_{0}\right)$ is the minimum pre fixed-point assignment of $v_{0}$.

We note that the termination argument is not completely straightforward as there is not a guarantee that it terminates within a polynomial number of 


\begin{tabular}{|c|c|c|c|c|c|c|c|c|}
\hline$i$ & $A(a)$ & $A(b)$ & $A(c)$ & $A(d)$ & $W$ & $D(b)$ & $D(c)$ & $D(d)$ \\
\hline 1 & $\infty$ & $\perp$ & $\perp$ & $\perp$ & $(a, 5, b)$ & & & \\
2 & $\infty$ & $\infty$ & $\perp$ & $\perp$ & $(b,\{(0, c),(3, d)\})$ & $(a, 5, b)$ & & \\
3 & $\infty$ & $\infty$ & $\infty$ & $\perp$ & $(c,\{(0, d)\})$ & $(a, 5, b)$ & $(b,\{(0, c),(3, d)\})$ & \\
4 & $\infty$ & $\infty$ & $\infty$ & $\infty$ & $(d, \emptyset)$ & $(a, 5, b)$ & $(b,\{(0, c),(3, d)\})$ & $(c,\{(0, d)\})$ \\
5 & $\infty$ & $\infty$ & $\infty$ & 0 & $(c,\{(0, d)\})$ & $(a, 5, b)$ & $(b,\{(0, c),(3, d)\})$ & $(c,\{(0, d)\})$ \\
6 & $\infty$ & $\infty$ & 0 & 0 & $(b,\{(0, c),(3, d)\})$ & $(a, 5, b)$ & $(b,\{(0, c),(3, d)\})$ & $(c,\{(0, d)\})$ \\
7 & $\infty$ & 3 & 0 & 0 & $(a, 5, b)$ & $(a, 5, b)$ & $(b,\{(0, c),(3, d)\})$ & $(c,\{(0, d)\})$ \\
8 & 0 & 3 & 0 & 0 & & $(a, 5, b)$ & $(b,\{(0, c),(3, d)\})$ & $(c,\{(0, d)\})$ \\
\hline
\end{tabular}

Table 1. Execution of Algorithm 2 on SDG from Figure 4(a)

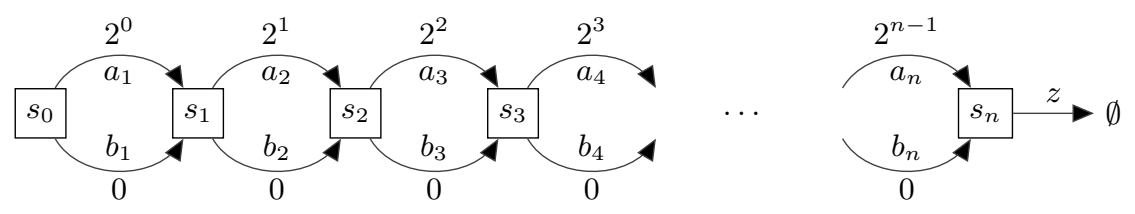

Fig. 5. A SDG where the local algorithm can take exponential running time

steps as depicted on the SDG in Figure 5 where for technical convenience, we named the hyper-edges $a_{1}, \ldots, a_{n}, b_{1}, \ldots, b_{n}$ and $z$. Consider now an execution of Algorithm 2 starting from the configuration $s_{0}$. Let us pick the edges from $W$ at line 4 according to the strategy:

- if $z \in W$ then pick $z$, else

- if $a_{i} \in W$ for some $i$ then pick $a_{i}$ (there will be at most one such $a_{i}$ ), else

- pick $b_{i} \in W$ with the smallest index $i$.

Then the initial assignment of $A\left(s_{0}\right)=\infty$ is gradually improved to $2^{n}-1,2^{n}-2$, $2^{n}-3, \ldots 1,0$. Hence, in the worst case, the local algorithm can perform exponentially many steps before it terminates, whereas the global algorithm always terminates in polynomial time. However, as we will see in Section 7, the local algorithm is in practice performing significantly better despite its high (theoretical) complexity.

\section{Model Checking with Symbolic Dependency Graphs}

We are now ready to present an encoding of a WKS and a WCTL formula as a symbolic dependency graph and hence decide the model checking problem via the computation of the minimum pre fixed-point assignment.

Given a WKS $\mathcal{K}$, a state $s$ of $\mathcal{K}$ and a WCTL formula $\varphi$, we construct the corresponding symbolic dependency graph as before with the exception that the existential and universal "until" operators are encoded by the rules given in Figure 6. 


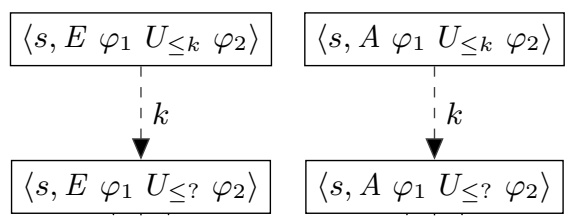

(a) Existential Until (b) Universal Until

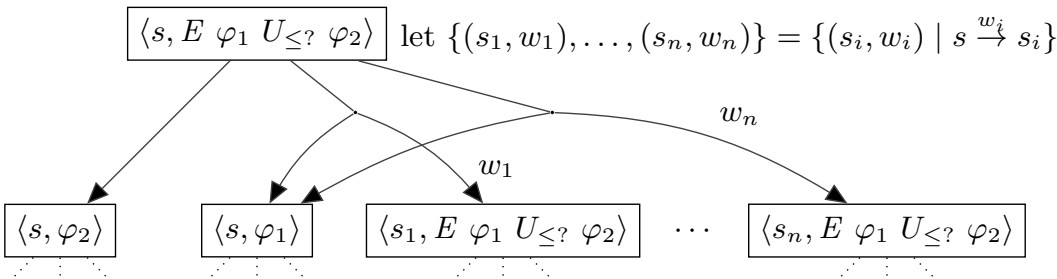

(c) Existential Until

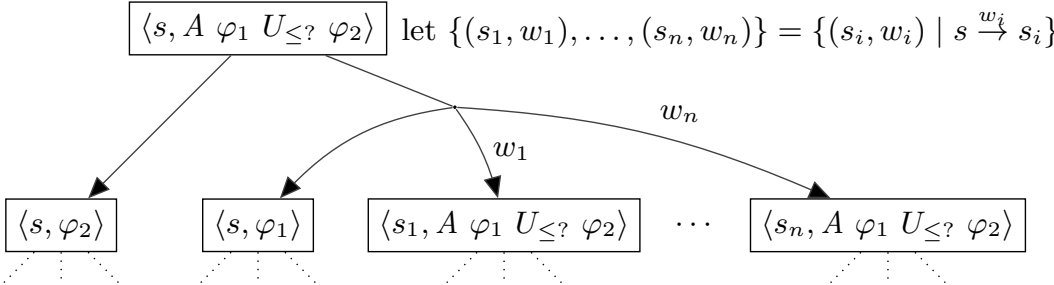

(d) Universal Until

Fig. 6. SDG encoding of existential and universal 'until' formulas

Theorem 5 (Encoding Correctness). Let $\mathcal{K}=(S, \mathcal{A P}, L, \rightarrow)$ be a WKS, $s \in S$ a state, and $\varphi$ a WCTL formula. Let $G$ be the constructed symbolic dependency graph rooted with $\langle s, \varphi\rangle$. Then $s \models \varphi$ if and only if $A_{\min }(\langle s, \varphi\rangle)=0$.

Proof. By structural induction on $\varphi$. Details are given in the appendix.

In Figure 7 we depict the symbolic dependency graph encoding of $E$ a $U_{\leq 1000} b$ for the configuration $s$ in the single-state WKS from Figure 3. This clearly illustrates the succinctness of SDG compared to standard dependency graphs. The minimum pre fixed-point assignment of this symbolic dependency graph is now reached in two iterations of the function $F$ defined in Equation (1).

We note that for a given WKS $\mathcal{K}=(S, \mathcal{A P}, L, \rightarrow)$ and a formula $\varphi$, the size of the constructed symbolic dependency graph $G=(V, H, C)$ can be bounded as follows: $|V|=O(|S| \cdot|\varphi|),|H|=O(|\rightarrow| \cdot|\varphi|)$ and $|C|=O(|\varphi|)$. In combination with Theorem 3 and the fact that $|C| \leq|H|$ (due to the rules for construction of $G$ ), we conclude with a theorem stating a polynomial time complexity of the global model checking algorithm for WCTL.

Theorem 6. Given a WKS $\mathcal{K}=(S, \mathcal{A P}, L, \rightarrow)$, a state $s \in S$ and a WCTL formula $\varphi$, the model checking problem $s \models \varphi$ is decidable in time $O\left(|S| \cdot|\rightarrow| \cdot|\varphi|^{3}\right)$. 


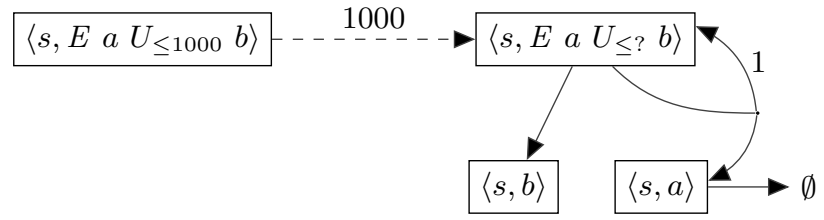

Fig. 7. SDG for the formula $s \models E a U_{\leq 1000} b$ and the WKS from Figure 3

As we already explained, the local model checking approach in Algorithm 2 may exhibit exponential running time. Nevertheless, the experiments in the section to follow show that this unlikely to happen in practice.

\section{$7 \quad$ Experiments}

In order to compare the performance of the algorithms for model checking WCTL, we developed a prototype tool implementation. There is a web-based front-end written in CoffeeScript available at

http://jonasfj.github.com/WKTool/

and the tool is entirely browser-based, requiring no installation. The model checking algorithms run with limited memory resources but the tool allows a fair comparison of the performance for the different algorithms. All experiments were conducted on a standard laptop (Intel Core i7) running Ubuntu Linux.

In order to experiment with larger, scalable models consisting of parallel components, we extend the process algebra CCS [18] with weight prefixing as well as proposition annotations and carry out experiments with weighted models of Leader Election [12], Alternating Bit Protocol [5], and Task Graph Scheduling problems for two processors [13]. The weight (communication cost) is associated with sending messages in the first two models while in the task graph scheduling the weight represents clock ticks of the processors.

\subsection{Dependency Graphs vs. Symbolic Dependency Graphs}

In Table 2 we compare the direct (standard dependency graph) algorithms with the symbolic ones. The execution times are in seconds and OOM indicates that verification runs out of memory. For a fixed size of the problems, we scale the bound $k$ in the WCTL formulae. In the leader election protocol with eight processes, we verified a satisfiable formula $E$ true $U_{\leq k}$ leader, asking if a leader can be determined within $k$ message exchanges, and an unsatisfiable formula $E$ true $U_{\leq k}$ leader $>1$, asking if there can be more than one leader selected within $k$ message exchanges. For the alternating bit protocol with a communication buffer of size four, we verified a satisfied formula $E$ true $U_{\leq k}$ delivered $=1$, asking if a message can be delivered within $k$ communication steps, and an unsatisfied formula $E$ true $U_{\leq k}\left(s_{0} \wedge d_{1}\right) \vee\left(s_{1} \wedge d_{0}\right)$, asking whether the sender and receiver can get out of synchrony withing the first $k$ communication steps. 


\begin{tabular}{|c|c|c|c|c|c|}
\hline \multicolumn{6}{|c|}{ Leader Election } \\
\hline & \multicolumn{2}{|c|}{ Direct } & \multicolumn{2}{|c|}{ Symbolic } & \\
\hline$k$ & Global & Local & Global & Local & \\
\hline 200 & 3.88 & 0.23 & 0.26 & 0.02 & I \\
\hline 400 & 8.33 & 0.25 & 0.26 & 0.02 & $\stackrel{\square}{*}$ \\
\hline 600 & OOM & 0.24 & 0.26 & 0.02 & 㔽 \\
\hline 800 & OOM & 0.25 & 0.26 & 0.02 & 2 \\
\hline 1000 & OOM & 0.26 & 0.27 & 0.02 & \\
\hline 200 & 7.76 & 8.58 & 0.26 & 0.26 & \\
\hline 400 & 17.05 & 20.23 & 0.26 & 0.26 & E \\
\hline 600 & OOM & OOM & 0.26 & 0.26 & 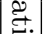 \\
\hline 800 & OOM & OOM & 0.26 & 0.26 & \\
\hline 1000 & OOM & OOM & 0.26 & 0.26 & 2 \\
\hline
\end{tabular}

\begin{tabular}{|c|c|c|c|c|c|}
\hline \multicolumn{6}{|c|}{ Alternating Bit Protocol } \\
\hline & \multicolumn{2}{|c|}{ Direct } & \multicolumn{2}{|c|}{ Symbolic } & \\
\hline$k$ & Global & Local & Global & Local & \\
\hline 100 & 3.87 & 0.05 & 0.23 & 0.03 & $\Omega$ \\
\hline 200 & 8.32 & 0.06 & 0.23 & 0.03 & $\stackrel{\square}{*}$ \\
\hline 300 & OOM & 0.10 & 0.28 & 0.04 & 惫 \\
\hline 400 & OOM & 0.11 & 0.23 & 0.03 & 2 \\
\hline 500 & OOM & 0.13 & 0.23 & 0.03 & \\
\hline 100 & 3.39 & 3.75 & 0.27 & 0.23 & -1 \\
\hline 200 & 6.98 & 8.62 & 0.30 & 0.25 & E \\
\hline 300 & OOM & 15.37 & 0.28 & 0.24 & 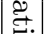 \\
\hline 400 & OOM & OOM & 0.27 & 0.24 & 量 \\
\hline 500 & OOM & OOM & 0.27 & 0.22 & 2 \\
\hline
\end{tabular}

Table 2. Scaling of bounds in WCTL formula (time in seconds)

For the satisfied formula, the direct global algorithm (global fixed-point computation on dependency graphs) runs out of memory as the bound $k$ in the formulae is scaled. The advantage of Liu and Smolka [16] local algorithm is obvious as on positive instances it performs (using DFS search strategy) about as well as the global symbolic algorithm. The local symbolic algorithm clearly performs best. We observed a similar behaviour also for other examples we tested and the symbolic algorithms were regularly performing better than the ones using the direct translation of WCTL formulae into dependency graphs. Hence we shall now focus on a more detailed comparison of the local vs. global symbolic algorithms.

\subsection{Local vs. Global Model Checking on SDG}

We shall now take a closer look at comparing the local and global symbolic algorithms. In Table 3 we return to the leader election and alternating bit protocol but we scale the sizes (number of processes and buffer capacity, resp.) of these models rather than the bounds in formulae. The satisfiable and unsatisfiable formulae are as before. In the leader election the verification of a satisfiable formula using the local symbolic algorithm is consistently faster as the instance size is incremented, while for unsatisfiable formulae the verification times are essentially the same. For the alternating bit protocol we present the results for the bound $k$ equal to 10,20 and $\infty$. While the results for unsatisfiable formulae do not change significantly, for the positive formula the bound 10 is very tight in the sense that there are only a few executions or "witnesses" that satisfy the formula. As the bound is relaxed, more solutions can be found which is reflected by the improved performance of the local algorithm, in particular in the situation where the upper-bound is $\infty$.

We also tested the algorithms on a larger benchmark of task graph scheduling problems [4]. The task graph scheduling problem asks about schedulability of a number of parallel tasks with given precedence constraints and processing 


\begin{tabular}{|c|c|c|c|}
\hline \multicolumn{4}{|c|}{ Leader Election } \\
\hline & \multicolumn{2}{|c|}{$k=200$} & \\
\hline$n$ & Global & Local & \\
\hline 7 & 0.08 & 0.01 & \\
\hline 8 & 0.26 & 0.02 & एم \\
\hline 9 & 1.06 & 0.03 & 点. \\
\hline 10 & 5.18 & 0.03 & $\stackrel{8}{2}$ \\
\hline 11 & 23.60 & 0.03 & \\
\hline 12 & Timeout & 0.04 & \\
\hline 7 & 0.08 & 0.08 & \\
\hline 8 & 0.26 & 0.26 & $\vec{b}$ \\
\hline 9 & 1.05 & 1.06 & 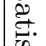 \\
\hline 10 & 4.97 & 4.96 & 票 \\
\hline 11 & 23.57 & 24.07 & 2 \\
\hline 12 & Timeout & Timeout & \\
\hline
\end{tabular}

\begin{tabular}{|c|c|c|c|c|c|c|c|}
\hline \multicolumn{8}{|c|}{ Alternating Bit Protocol } \\
\hline & \multicolumn{2}{|c|}{$k=10$} & \multicolumn{2}{|c|}{$k=20$} & \multicolumn{2}{|c|}{$k=\infty$} & \\
\hline$n$ & Global & Local & Global & Local & Global & Local & \\
\hline 5 & 0.33 & 0.10 & 0.33 & 0.07 & 0.33 & 0.04 & \\
\hline 6 & 0.78 & 0.18 & 0.77 & 0.17 & 0.80 & 0.06 & 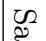 \\
\hline 7 & 1.88 & 0.34 & 1.92 & 0.14 & 1.96 & 0.05 & 宓. \\
\hline 8 & 4.82 & 0.82 & 4.71 & 0.72 & 4.78 & 0.09 & $\vec{\nabla}$ \\
\hline 9 & 13.91 & 10.60 & 12.41 & 1.67 & 12.92 & 0.20 & \\
\hline 10 & OOM & OOM & OOM & 6.29 & OOM & 0.23 & \\
\hline 4 & 0.27 & 0.24 & 0.27 & 0.23 & 0.29 & 0.24 & 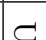 \\
\hline 5 & 0.54 & 0.43 & 0.51 & 0.37 & 0.57 & 0.40 & $\vec{b}$ \\
\hline 6 & 1.42 & 0.98 & 1.21 & 0.93 & 1.31 & 1.02 & . \\
\hline 7 & 2.70 & 2.05 & 2.93 & 2.06 & 3.14 & 2.21 & 急 \\
\hline 8 & 6.15 & 4.98 & 7.08 & 5.57 & 6.86 & 5.34 & 2 \\
\hline 9 & OOM & OOM & OOM & OOM & OOM & OOM & \\
\hline
\end{tabular}

Table 3. Scaling the model size for the symbolic algorithms (time in seconds)

times that are executed on a fixed number of homogeneous processors [13]. We automatically generate models for two processors from the benchmark containing in total 180 models and scaled them by the number of initial tasks that we include from each case into schedulability analysis.

The first three task graphs (T0, T1 and T2) are presented in Table 4. We model check nested formulae and the satisfiable one is $E$ true $U_{\leq 90}\left(t_{n-2}^{\text {ready }} \wedge\right.$ $A$ true $U_{\leq 80}$ done) asking whether there is within 500 clock ticks a configuration where the task $t_{n-2}$ can be scheduled such that then we have a guarantee that the whole schedule terminates within 500 ticks. When the upper-bounds are decreased to 5 and 10 the formula becomes unsatisfiable for all task graphs in the benchmark.

Finally, we verify the formula $E$ true $U_{\leq k}$ done asking whether the task graph can be scheduled within $k$ clock ticks. We run the whole benchmark through the test (180 cases) for values of $k$ equal to 30,60 and 90 , measuring the number of finished verification tasks (without running out of resources) and the total accumulated time it took to verify the whole benchmark for those cases where both the global and local algorithms provided an answer. The results are listed in Table 5. This provides again an evidence for the claim that the local algorithm profits from the situation where there are more possible schedules as the bound $k$ is being relaxed.

\section{Conclusion}

We suggested a symbolic extension of dependency graphs in order to verify negation-free weighted CTL properties where temporal operators are annotated with upper-bound constraints on the accumulated weight. Then we introduced global and local algorithms for the computation of fixed-points in order to answer 


\begin{tabular}{|c|c|c|c|c|c|c|c|}
\hline & \multicolumn{2}{|c|}{ T0 } & \multicolumn{2}{|c|}{$\mathrm{T} 1$} & \multicolumn{2}{|c|}{$\mathrm{T} 2$} & \\
\hline$n$ & Global & Local & Global & Local & Global & Local & \\
\hline 2 & 0.24 & 0.04 & 0.06 & 0.01 & 0.07 & 0.01 & \\
\hline 3 & 3.11 & 0.01 & 0.15 & 0.08 & 0.19 & 0.01 & \\
\hline 4 & 4.57 & 1.13 & 0.18 & 0.08 & 0.88 & 0.19 & $\Omega$ \\
\hline 5 & 6.09 & 0.03 & 2.73 & 0.01 & 7.05 & 0.02 & $\stackrel{\text { I. }}{=}$ \\
\hline 6 & OOM & OOM & 5.27 & 1.08 & OOM & 1.44 & $\stackrel{5}{2}$ \\
\hline 7 & OOM & 0.02 & $\mathrm{OOM}$ & 0.02 & OOM & 0.01 & 2 \\
\hline 8 & OOM & 0.03 & $\mathrm{OOM}$ & OOM & OOM & 2.75 & \\
\hline 9 & OOM & OOM & OOM & OOM & OOM & 1.86 & \\
\hline 10 & OOM & 0.03 & OOM & OOM & OOM & OOM & \\
\hline 2 & 0.22 & 0.20 & 0.05 & 0.05 & 0.08 & 0.01 & $\subset$ \\
\hline 3 & 2.91 & 2.55 & 0.14 & 0.13 & 0.20 & 0.01 & $\vec{b}$ \\
\hline 4 & 6.35 & 4.45 & 0.16 & 0.14 & 0.91 & 0.20 & $\stackrel{\text { ڤ્. }}{\rightleftarrows}$ \\
\hline 5 & 7.45 & 5.00 & 2.31 & 1.69 & 7.48 & 0.03 & $\stackrel{2}{9}$ \\
\hline 6 & OOM & OOM & 4.67 & 4.40 & OOM & 1.40 & 2 \\
\hline 7 & OOM & OOM & OOM & OOM & OOM & OOM & \\
\hline
\end{tabular}

Table 4. Scaling task graphs by the number of initial tasks (time is seconds)

\begin{tabular}{|l||r|r|r|r|r|r|}
\hline 180 task graphs for & \multicolumn{2}{|c|}{$k=30$} & \multicolumn{2}{|c|}{$k=60$} & \multicolumn{2}{|c|}{$k=90$} \\
\hline Algorithm & global & local & global & local & global & local \\
\hline \hline Number of finished tasks & 32 & 85 & 32 & 158 & 32 & 178 \\
Accumulated time (seconds) & 50.4 & 12.9 & 47.6 & 2.30 & 47.32 & 0.44 \\
\hline
\end{tabular}

Table 5. Summary of task graphs verification (180 cases in total)

the model checking problems for the logic. The algorithms were implemented and experimented with, coming to the conclusion that the local symbol algorithm is the preferred one, providing order of magnitude speedup in the cases where the bounds in the logical formula allow for a larger number of possible witnesses of satisfiability of the formula.

In the future work we will study a weighted CTL logic with negation that combines lower- and upper-bounds. (The model checking problem for a logic containing weight intervals as the constraints is already NP-hard; see appendix for a straightforward proof of this.) From the practical point of view it would be worth designing good heuristics that can guide the search in the local algorithm in order to find faster the witnesses of satisfiability of a formula. Another challenging problem is to adapt our technique to support alternating fixed points.

\section{References}

1. Rajeev Alur and David L. Dill. Automata for modeling real-time systems. In Mike Paterson, editor, ICALP, volume 443 of Lecture Notes in Computer Science, pages 322-335. Springer, 1990. 
2. Rajeev Alur, Salvatore La Torre, and George J. Pappas. Optimal paths in weighted timed automata. In Benedetto and Sangiovanni-Vincentelli [7], pages 49-62.

3. Henrik Reif Andersen. Model checking and boolean graphs. Theoretical Computer Science, 126(1):3 - 30, 1994.

4. Kasahara Laboratory at Waseda University. Standard task graph set. http://www.kasahara.elec.waseda.ac.jp/schedule/.

5. K. A. Bartlett, R. A. Scantlebury, and P. T. Wilkinson. A note on reliable full-duplex transmission over half-duplex links. Communications of the ACM, 12(5):260-261, 1969.

6. Gerd Behrmann, Ansgar Fehnker, Thomas Hune, Kim Guldstrand Larsen, Paul Pettersson, Judi Romijn, and Frits W. Vaandrager. Minimum-cost reachability for priced timed automata. In Benedetto and Sangiovanni-Vincentelli [7], pages 147-161.

7. Maria Domenica Di Benedetto and Alberto L. Sangiovanni-Vincentelli, editors. Hybrid Systems: Computation and Control, 4th International Workshop, HSCC 2001, Rome, Italy, March 28-30, 2001, Proceedings, volume 2034 of Lecture Notes in Computer Science. Springer, 2001.

8. Patricia Bouyer, Kim Guldstrand Larsen, and Nicolas Markey. Model checking one-clock priced timed automata. Logical Methods in Computer Science, 4(2), 2008.

9. Thomas Brihaye, Véronique Bruyère, and Jean-François Raskin. Model-checking for weighted timed automata. In Yassine Lakhnech and Sergio Yovine, editors, FORMATS/FTRTFT, volume 3253 of Lecture Notes in Computer Science, pages 277-292. Springer, 2004.

10. Peter Buchholz and Peter Kemper. Model checking for a class of weighted automata. Discrete Event Dynamic Systems, 20:103-137, 2010.

11. Franck Cassez, Alexandre David, Emmanuel Fleury, Kim G. Larsen, and Didier Lime. Efficient on-the-fly algorithms for the analysis of timed games. In $I N$ CONCUR 05, LNCS 3653, pages 66-80. Springer, 2005.

12. E. Chang and R. Roberts. An improved algorithm for decentralized extrema-finding in circular configurations of processes. Commun. of ACM, 22(5):281-283, 1979.

13. Y.-K. Kwok and I. Ahmad. Benchmarking and comparison of the task graph scheduling algorithms. Journal of Parallel and Distributed Computing, 59(3):381 - 422, 1999.

14. François Laroussinie, Nicolas Markey, and Ghassan Oreiby. Model-checking timed atl for durational concurrent game structures. In Eugene Asarin and Patricia Bouyer, editors, FORMATS, volume 4202 of Lecture Notes in Computer Science, pages 245-259. Springer, 2006.

15. Xinxin Liu, C.R. Ramakrishnan, and ScottA. Smolka. Fully local and efficient evaluation of alternating fixed points. In Tools and Algorithms for the Construction and Analysis of Systems, volume 1384 of LNCS, pages 5-19. Springer Berlin Heidelberg, 1998.

16. Xinxin Liu and Scott A. Smolka. Simple linear-time algorithms for minimal fixed points (extended abstract). In ICALP, pages 53-66, 1998.

17. Silvano Martello and Paolo Toth. Knapsack problems: algorithms and computer implementations. John Wiley \& Sons, Inc., New York, NY, USA, 1990.

18. R. Milner. A calculus of communicating systems. LNCS, 92, 1980. 


\section{A Appendix}

\section{A.1 Proofs related to dependency graphs}

\section{Proof of Theorem 2}

Let $\mathcal{K}=(S, \mathcal{A P}, L, \rightarrow)$ be a WKS, $s \in S$ a state, $\varphi$ a WCTL formula. Let $G$ be the constructed dependency graph rooted with $\langle s, \varphi\rangle$. Then $s \models \varphi$ if and only if $A_{\min }(\langle s, \varphi\rangle)=1$.

Proof. We prove Theorem 2 by structural induction on $\varphi$.

(I) For $\varphi=$ true we show that for all $s \in S$ we have $A_{\min }(\langle s$, true $\rangle)=1$ if and only if $s \models$ true. But as $s \models$ true always holds, it is sufficient to show that $A_{\min }(\langle s$, true $\rangle)=1$ for any pre fixed-point assignment $A$ of $G$. In Figure 2(a) we add a hyper-edge from the configuration $\langle s$, true $\rangle$, to the empty target set. Thus, we have that $A(v)=1$ for any pre fixedpoint assignment $A$ of $G$, because all vertices in the empty set satisfy any property vacuously.

(II) For $\varphi=a$ we prove that $A_{\min }(\langle s, a\rangle)=1$ if and only if $s \models a$ for all $s \in S$. If $a \in L(s)$ we have $s \models a$ and by Figure 2(b), there is a hyper-edge from the configuration $\langle s, a\rangle$ to the empty target set. As in (I) this means that $A_{\min }(\langle s, a\rangle)=1$, which leaves us to consider $a \notin L(s)$. In this case we obviously have $s \not \models a$ and by the side-condition in Figure 2(b), we can conclude that there is no hyper-edge from the configuration $\langle s, a\rangle$ when $a \notin L(s)$. Thus, we have $A_{\min }(\langle s, a\rangle)=0$ because $A_{\min }$ is the minimum pre fixed-point assignment.

(III) For $\varphi=\varphi_{1} \wedge \varphi_{2}$ we show that $A_{\min }\left(\left\langle s, \varphi_{1} \wedge \varphi_{2}\right\rangle\right)=1$ if and only if $s \models \varphi_{1} \wedge \varphi_{2}$ for all $s \in S$. By Figure 2(c), a configuration $\left\langle s, \varphi_{1} \wedge \varphi_{2}\right\rangle$ has a single hyper-edge with the target set $\left\{\left\langle s, \varphi_{1}\right\rangle,\left\langle s, \varphi_{2}\right\rangle\right\}$. With this observation it is easy to see that $A_{\min }\left(\left\langle s, \varphi_{1} \wedge \varphi_{2}\right\rangle\right)=1$ if and only if $A_{\min }\left(\left\langle s, \varphi_{1}\right\rangle\right)=1$ and $A_{\min }\left(\left\langle s, \varphi_{2}\right\rangle\right)=1$. By the induction hypothesis this is equivalent to $s \models \varphi_{1}$ and $s \models \varphi_{2}$, which following the semantics implies $s=\varphi_{1} \wedge \varphi_{2}$.

(IV) For $\varphi=\varphi_{1} \vee \varphi_{2}$ we show that $A_{\min }\left(\left\langle s, \varphi_{1} \vee \varphi_{2}\right\rangle\right)=1$ if and only if $s \models \varphi_{1} \vee$ $\varphi_{2}$ for all $s \in S$. By Figure 2(d), a configuration $\left\langle s, \varphi_{1} \wedge \vee_{2}\right\rangle$ has two hyperedges with the target sets $\left\{\left\langle s, \varphi_{1}\right\rangle\right\}$ and $\left\{\left\langle s, \varphi_{2}\right\rangle\right\}$. With this observation, we have that $A_{\min }\left(\left\langle s, \varphi_{1} \vee \varphi_{2}\right\rangle\right)=1$ if and only if $A_{\min }\left(\left\langle s, \varphi_{1}\right\rangle\right)=1$ or $A_{\min }\left(\left\langle s, \varphi_{2}\right\rangle\right)=1$. By the induction hypothesis this is equivalent to $s \models \varphi_{1}$ or $s \models \varphi_{2}$, which following the semantics implies $s=\varphi_{1} \vee \varphi_{2}$.

(V) For $\varphi=E \varphi_{1} U_{\leq k} \varphi_{2}$ we show that $A_{\min }\left(\left\langle s, E \varphi_{1} U_{\leq k} \varphi_{2}\right\rangle\right)=1$ if and only if $s \models E \varphi_{1} U_{\leq k} \varphi_{2}$ for all $s \in S$. Recall the semantics for the satisfaction of formula $E \varphi_{1} U_{\leq k} \varphi_{2}$, requires that for some $k^{\prime} \leq k$, there exists a run $\sigma$ and a position $p \geq 0$ that satisfy the following conditions.

$$
\begin{aligned}
\sigma(p) & \models \varphi_{2} \\
\sigma(j) & \models \varphi_{1}, \text { for all } j<p \\
W_{\sigma}(p) & \leq k^{\prime}
\end{aligned}
$$


$\Rightarrow$ : Assume that $A_{\min }\left(\left\langle s, E \varphi_{1} U_{\leq k} \varphi_{2}\right\rangle\right)=1$, we now show that this implies $s \models E \varphi_{1} U_{\leq k} \varphi_{2}$.

We denote the iteration in which a configuration $v$ was first assigned the value 1 , as $Z(v)$, formally we write the auxiliary function $Z$ as follows.

$$
Z(v)= \begin{cases}i & \text { if } F^{i}\left(A_{0}\right)(v) \neq F^{i-1}\left(A_{0}\right)(v) \\ \infty & \text { otherwise }\end{cases}
$$

For any configuration $v$ it holds that $Z(v)<\infty$ if and only if $A_{\min }(v)=1$, as a pre fixed-point assignment must be reached in a finite number of iterations. Considering $Z(v)$ for a configuration $v=\left\langle s, E \varphi_{1} U_{\leq k} \varphi_{2}\right\rangle$, where $A_{\min }(v)=1$, we see that in iteration $Z(v)-1$, the assignment of some configuration in the target-set for a hyper-edge to $v$ must have been changed to 1. In Figure 2(e) we observe that there are two kinds of hyper-edges, leading us to conclude that at least one of the following two cases must hold.

A) $Z\left(\left\langle s, \varphi_{2}\right\rangle\right)=Z(v)-1$, or

B) $\max \left\{Z\left(\left\langle s, \varphi_{1}\right\rangle\right), Z\left(\left\langle s^{\prime}, E \varphi_{1} U_{\leq k-w} \varphi_{2}\right\rangle\right)\right\}=Z(v)-1$, for some $s^{\prime}$, s.t. $s \stackrel{w}{\rightarrow} s^{\prime}$.

We now show that $A_{\min }\left(\left\langle s, E \varphi_{1} U_{\leq k} \varphi_{2}\right\rangle\right)=1$ implies the existence of a run $\sigma$ and a position $p$ satisifying conditions 2,3 and 4 for $k^{\prime} \leq k$, by induction on $Z\left(\left\langle s, E \varphi_{1} U_{\leq k} \varphi_{2}\right\rangle\right)$.

First we observe that $Z\left(\left\langle s, E \varphi_{1} U_{\leq k} \varphi_{2}\right\rangle\right)$ is always greater than 1 , as only configurations $v$ having trivial hyper-edges $(v, \emptyset)$ are assigned 1 in the first iteration of $F$.

Base Case $\left(Z\left(\left\langle s, E \quad \varphi_{1} U_{\leq k} \varphi_{2}\right\rangle\right)=2\right)$ : In this case we know that case (A) must hold, seeing that no configuration $u=\left\langle s^{\prime}, E \varphi_{1} U_{\leq k-w} \varphi_{2}\right\rangle$ can have $Z(u)=1$. From case (A), we have that $Z\left(\left\langle s, \varphi_{2}\right\rangle\right)=1$, which means that $A_{\min }\left(\left\langle s, \varphi_{2}\right\rangle\right)=1$. By structural induction, $A_{\min }\left(\left\langle s, \varphi_{2}\right\rangle\right)=1$ gives us $s \models \varphi_{2}$. Thus, any run $\sigma=s \ldots$ and position $p=0$ satisfy conditions 2, 3 and 4 for $k^{\prime}=0$, hence, it also holds for $k^{\prime} \leq k$.

Inductive Step $\left(Z\left(\left\langle s, E \varphi_{1} U_{\leq k} \varphi_{2}\right\rangle\right)>2\right)$ : Again, we consider cases (A) and (B). If case (A) holds we can construct a run $\sigma=s \ldots$ and position $p=0$ as before. If (B) is the case, we have that $A_{\min }\left(\left\langle s, \varphi_{1}\right\rangle\right)=1$ and $A_{\min }\left(\left\langle s^{\prime}, E \varphi_{1} U_{\leq k-w} \varphi_{2}\right\rangle\right)=1$. By structural induction it follows from $A_{\text {min }}\left(\left\langle s, \varphi_{1}\right\rangle\right)=1$ that $s \models \varphi_{1}$.

Because $Z\left(\left\langle s^{\prime}, E \quad \varphi_{1} U_{\leq k-w} \varphi_{2}\right\rangle\right)<Z\left(\left\langle s, E \varphi_{1} U_{\leq k} \varphi_{2}\right\rangle\right)$ it follows by induction that there is a run $\sigma=s^{\prime} \ldots$ and a position $p$ that satisfy conditions 2, 3 and 4 for $k^{\prime} \leq k-w$. Considering the extension $\sigma^{\prime}=s \stackrel{w}{\rightarrow}$ $s^{\prime} \ldots$ of $\sigma$ and position $p^{\prime}=p+1$, we observe that $\sigma^{\prime}$ and $p^{\prime}$ also satisfy the conditions for $k^{\prime} \leq k$.

- Condition 2 holds because $\sigma^{\prime}\left(p^{\prime}\right)=\sigma(p)$ and $\sigma(p) \models \varphi_{2}$.

- Condition 3 holds since $\sigma(0)=s, s \models \varphi_{1}$ and for all $j<p$ we have $\sigma^{\prime}(j+1)=\sigma(j)$ and $\sigma(j) \models \varphi_{1}$.

- Condition 4 holds due to the fact that $W_{\sigma}(p) \leq k-w$ implies $W_{\sigma^{\prime}}\left(p^{\prime}\right) \leq$ $k$, because $W_{\sigma^{\prime}}\left(p^{\prime}\right)-W_{\sigma}(p)=w$. 
We have now shown that $A_{\min }\left(\left\langle s, E \varphi_{1} U_{\leq k} \varphi_{2}\right\rangle\right)=1$ implies that there exists a run $\sigma$ starting from $s$ and a position $p$ satisfying conditions 2,3 and 4 for $k^{\prime} \leq k$. Thus, given the semantics it follows that $s \models E \varphi_{1} U_{\leq k} \varphi_{2}$. $\Leftarrow$ : Assume that $s \models E \varphi_{1} U_{\leq k} \varphi_{2}$, we now show that this implies $A_{\min }\left(\left\langle s, E \quad \varphi_{1} U_{\leq k} \varphi_{2}\right\rangle\right)=1$. From the semantics it follows that there is a run $\sigma$ and position $p$ satisfying conditions 2,3 and 4 for $k^{\prime} \leq k$ Let $s=s_{0}$, then we can write $\sigma$ as follows.

$$
\sigma=s_{0} \stackrel{w_{1}}{\rightarrow} s_{1} \ldots s_{p-1} \stackrel{w_{p}}{\rightarrow} s_{p} \ldots
$$

We show that $A_{\min }\left(\left\langle s_{i}, E \varphi_{1} U_{\leq k-W_{\sigma}(i)} \varphi_{2}\right\rangle\right)=1$ by induction on $i$ starting from $p$.

Base Case $(i=p)$ : By condition 2 of the semantics, $s_{p}=\varphi_{2}$, which by structural induction on $\varphi$ implies $A_{\min }\left(\left\langle s_{p}, \varphi_{2}\right\rangle\right)=1$. In Figure 2(e), we observe that there is a hyper-edge from $\left\langle s_{p}, E \varphi_{1} U_{\leq k-W_{\sigma}(i)} \varphi_{2}\right\rangle$ to $\left\langle s_{p}, \varphi_{2}\right\rangle$, thus, $A_{\min }\left(\left\langle s_{p}, \varphi_{2}\right\rangle\right)=1$ implies $A_{\min }\left(\left\langle s_{p}, E \varphi_{1} \bar{U}_{\leq k-W_{\sigma}(i)} \varphi_{2}\right\rangle\right)=$ 1 , which proves our base case.

Inductive Step $(i<p)$ : By condition 3 of the semantics, $s_{i} \models \varphi_{1}$, which by structural induction on $\varphi$ implies $A_{\min }\left(\left\langle s_{i}, \varphi_{1}\right\rangle\right)=1$. By induction on $i$, we know that $A_{\min }\left(\left\langle s_{i+1}, E \varphi_{1} U_{\leq k-W_{\sigma}(i+1)} \varphi_{2}\right\rangle\right)=1$ holds. In Figure $2(\mathrm{e})$, we observe that there is a hyper-edge $e$ from $\left\langle s_{i}, E \varphi_{1} U_{\leq k-W_{\sigma}(i)} \varphi_{2}\right\rangle$ to the target-set $\left\langle s_{i}, \varphi_{1}\right\rangle$ and $\left\langle s_{i+1}, E \varphi_{1} U_{\leq k-W_{\sigma}(i+1)} \varphi_{2}\right\rangle$, as $W_{\sigma}(i+1)-$ $W_{\sigma}(i)=w_{i+1}$, which is exactly the transition weight between $s_{i}$ and $s_{i+1}$. Since we know that $A_{\min }(v)=1$ for all configurations $v$ of the target-set of the hyper-edge $e$, then it must follow that $A_{\min }\left(\left\langle s_{i}, E \varphi_{1} U_{\leq k-W_{\sigma}(i)} \varphi_{2}\right\rangle\right)=$ 1 for all $i \leq p$.

(VI) For $\varphi=A \varphi_{1} U_{\leq k} \varphi_{2}$ we have that $A_{\min }\left(\left\langle s, A \varphi_{1} U_{\leq k} \varphi_{2}\right\rangle\right)=1$ if and only if $s=A \varphi_{1} U_{\leq k} \varphi_{2}$ for all $s \in S$. Recall the semantics for the satisfaction of formula $A \varphi_{1} U_{\leq k} \varphi_{2}$, requires that for any run $\sigma$ there exists a position $p \geq 0$ satisfying the following conditions for $k^{\prime} \leq k$.

$$
\begin{aligned}
\sigma(p) & \models \varphi_{2} \\
\sigma(j) & \models \varphi_{1}, \text { for all } j<p \\
W_{\sigma}(p) & \leq k^{\prime}
\end{aligned}
$$

$\Rightarrow$ : Assume that $A_{\min }\left(\left\langle s, A \varphi_{1} U_{\leq k} \varphi_{2}\right\rangle\right)=1$, we now show that this implies $s \models A \varphi_{1} U_{\leq k} \varphi_{2}$.

We denote the iteration in which a configuration $v$ was first assigned 1 , as $Z(v)$, formally we write the auxiliary function $Z$ as in Equation 5, shown in the previous case.

For any configuration $v$ it holds that $Z(v)<\infty$ if and only if $A_{\min }(v)=1$, as a pre fixed-point assignment must be reached in a finite number of iterations. Considering $Z(v)$ for a configuration $v=\left\langle s, A \varphi_{1} U_{\leq k} \varphi_{2}\right\rangle$, where $A_{\min }(v)=1$, we see that in iteration $Z(v)-1$, the assignment of some configuration in the target-set for a hyper-edge to $v$ must have been changed to 1 . In Figure 2(f) we see that there are at most two hyper-edges, 
leading us to conclude that at least one of the following two cases must hold.
A) $Z\left(\left\langle s, \varphi_{2}\right\rangle\right)=Z(v)-1$, or
B) $\mathrm{Z}(\mathrm{v})-1=\max \left\{\begin{array}{l}Z\left(\left\langle s, \varphi_{1}\right\rangle\right) \\ Z\left(\left\langle s^{\prime}, A \varphi_{1} U_{\leq k-w} \varphi_{2}\right\rangle\right) \quad \text { for all } s^{\prime} \text {, s.t. } s \stackrel{w}{\rightarrow} s^{\prime}\end{array}\right.$

For any configuration $v=\left\langle s, A \varphi_{1} U_{\leq k} \varphi_{2}\right\rangle$, we now show by induction on $Z(v)$ that $A_{\min }(v)=1$ implies that for any run $\sigma=s \ldots$, there is a position $p$ satisfying conditions 6,7 and 8 for $k^{\prime} \leq k$. We observe that $Z(v)$ is always greater than 1 , seeing that $v$ does not have a trivial hyper-edge $(v, \emptyset)$, and only configurations with trivial hyper-edges are assigned the value 1 in $F^{1}$.

Base Case $\left(Z\left(\left\langle s, A \varphi_{1} U_{\leq k} \varphi_{2}\right\rangle\right)=2\right)$ : It must be the case that (A) holds, as it is not possible for any configuration on the form $u=\left\langle s^{\prime}, A \varphi_{1} U_{\leq k-w} \varphi_{2}\right\rangle$ to have $Z(u)=1$. From case (A), we have that $Z\left(\left\langle s, \varphi_{2}\right\rangle\right)=1$ which implies that $A_{\min }\left(\left\langle s, \varphi_{2}\right\rangle\right)=1$. Hence, by structural induction it follows that $s=\varphi_{2}$. For any run $\sigma=s \ldots$ we have that $p=0$ is a position that satisfies conditions 6,7 and 8 for $k^{\prime} \leq k$.

Inductive Step $\left(Z\left(\left\langle s, A \varphi_{1} U_{\leq k} \varphi_{2}\right\rangle\right)>2\right)$ : Once more, we consider cases (A) and (B). If case (A) holds then for any run $\sigma=s \ldots$ we have position $p=0$ that satisifes the conditions as before. If (B) is the case, we have that $A_{\min }\left(\left\langle s, \varphi_{1}\right\rangle\right)=1$ and for all $s_{i}$ s.t. $s \stackrel{w_{i}}{\rightarrow} s_{i}$, it holds that $A_{\min }\left(\left\langle s_{i}, A \varphi_{1} U_{\leq k-w_{i}} \varphi_{2}\right\rangle\right)=1$, which by induction on $Z\left(\left\langle s_{i}, A \varphi_{1} U_{\leq k-w_{i}} \varphi_{2}\right\rangle\right)$ implies that $s_{i} \models\left\langle s_{i}, A \varphi_{1} U_{\leq k-w_{i}} \varphi_{2}\right\rangle$. By structural induction it follows from $A_{\min }\left(\left\langle s, \varphi_{1}\right\rangle\right)=1$ that $s=\varphi_{1}$.

Considering any run $\sigma$ starting from $s$, we see that this run must be on the form $\sigma=s \stackrel{w_{i}}{\rightarrow} s_{i} \ldots$ for some $s_{i}$, s.t. $s \stackrel{w_{i}}{\rightarrow} s_{i}$. For any postfix $\sigma^{\prime}=s_{i} \ldots$ of $\sigma$, there exists a position $p^{\prime}$ satisfying conditions 6,7 and 8 for $k^{\prime} \leq k-w_{i}$, as $s_{i} \models A \varphi_{1} U_{\leq k-w_{i}} \varphi_{2}$. Thus, given $\sigma$ we have that $p=p^{\prime}+1$ is a position satisfying conditions 6,7 and 8 for $k^{\prime} \leq k$.

- Condition 6 holds because $\sigma(p)=\sigma^{\prime}\left(p^{\prime}\right)$ and $\sigma^{\prime}\left(p^{\prime}\right) \mid=\varphi_{2}$.

- Condition 7 holds since $\sigma(0)=s, s \mid=\varphi_{1}$ and for all $j<p^{\prime}$ we have $\sigma(j+1)=\sigma^{\prime}(j)$ and $\sigma^{\prime}(j) \models \varphi_{1}$.

- Condition 8 holds due to the fact that $W_{\sigma}^{\prime}\left(p^{\prime}\right) \leq k-w_{i}$ implies $W_{\sigma}(p) \leq k$, because $W_{\sigma}(p)-W_{\sigma}^{\prime}\left(p^{\prime}\right)=w_{i}$.

We have now shown that $A_{\min }\left(\left\langle s, A \varphi_{1} U_{\leq k} \varphi_{2}\right\rangle\right)=1$ implies that for any run $\sigma$ starting from $s$, there is a position $p$ satisfying conditions 6,7 and 8 for $k^{\prime} \leq k$. Thus, it follows from the semantics that $s=A \varphi_{1} U_{\leq k} \varphi_{2}$. $\Leftarrow$ : Assume that $s=A \varphi_{1} U_{\leq k} \varphi_{2}$, we now show that this implies $A_{\min }\left(\left\langle s, A \varphi_{1} U_{\leq k} \varphi_{2}\right\rangle\right)=1$.

Considering the formula $\varphi=A \varphi_{1} U_{\leq k} \varphi_{2}$ and state $s$, if $s \models \varphi$ then it follows from the semantics that for any run $\sigma$ starting from $s$, there is a position $p$ that satisfies conditions 6,7 and 8 for $k^{\prime} \leq k$. Given $\sigma=s \ldots$, the existence of $p$ also implies the existence of some smallest $p^{\prime}$. By $\rho(s, \varphi)$, 
we denote maximum such smallest $p^{\prime}$ for any run starting from $s$.

$$
\rho\left(s, A \varphi_{1} U_{\leq k} \varphi_{2}\right)=\max \left\{\begin{array}{l}
\text { smallest } p \text { satisfying } \\
6,7 \text { and } 8 \text { for } k^{\prime} \leq k
\end{array} \mid \text { for all } \sigma=s \ldots\right\}
$$

Considering the state $s$ and formula $\varphi=A \varphi_{1} U_{\leq k} \varphi_{2}$, we now show that $s=\varphi$ implies $A_{\min }(\langle s, \varphi\rangle)=1$ by induction on $\rho(s, \varphi)$.

Base Case $(\rho(s, \varphi)=0)$ : In this case we have that for any run $\sigma=s \ldots$, the position $p=0$ satisifies conditions 6,7 and 8 for $k^{\prime} \leq k$. Condition 6 implies that $s=\varphi_{2}$ which by structural induction implies $A_{\min }\left(\left\langle s, \varphi_{2}\right\rangle\right)=$ 1. In Figure 2(f) we see that $\langle s, \varphi\rangle$ has a hyper-edge to $\left\langle s, \varphi_{2}\right\rangle$. Thus, it must hold that $A_{\min }(\langle s, \varphi\rangle)=1$.

Inductive Step $(\rho(s, \varphi)>0)$ : In this case we have that for any run $\sigma=s \ldots$, there is a position $p \leq \rho(s, \varphi)$ which satisifies conditions 6,7 and 8 for $k^{\prime} \leq k$. We also know that $p>0$, because if $p$ were 0 for some run $\sigma=s \ldots$, then this would imply $s \models \varphi_{2}$, in which case the smallest $p$ would be 0 for any run $\sigma=s \ldots$. Thus, as $\rho(s, \varphi)>0$ this cannot be the case and $p>0$, which from condition 7 implies that $s \models \varphi_{1}$ and by structural induction we have that $A_{\min }\left(\left\langle s, \varphi_{1}\right\rangle\right)=1$.

In Figure 2(f) we see that $\langle s, \varphi\rangle$ has a hyper-edge to the target-set containing $\left\langle s, \varphi_{1}\right\rangle$ and $\left\langle s_{i}, A \varphi_{1} U_{\leq k-w_{i}} \varphi_{2}\right\rangle$ for all $s_{i}$ s.t. $s \stackrel{w_{i}}{\rightarrow} s_{i}$. Thus, to show that $A_{\min }(\langle s, \varphi\rangle)=1$ we need only show that $A_{\min }\left(\left\langle s_{i}, A \varphi_{1} U_{\leq k-w_{i}} \varphi_{2}\right\rangle\right)=$ 1 for all $s_{i}$ s.t. $s \stackrel{w_{i}}{\rightarrow} s_{i}$.

Consider some $s_{i}$ s.t. $s \stackrel{w_{i}}{\rightarrow} s_{i}$, then any run $\sigma^{\prime}=s_{i} \ldots$ starting from $s_{i}$ must be a postfix of some run $\sigma=s \stackrel{w_{i}}{\rightarrow} s_{i} \ldots$ starting from $s$. We know that given $\sigma$, there exists a position $p \leq \rho(s, \varphi)$ satisifying conditions 6 , 7 and 8 for $k^{\prime} \leq k$. Now considering $\sigma^{\prime}$ we have that position $p^{\prime}=p-1$ also satisifies these conditions for $k^{\prime} \leq k-w_{i}$.

- Condition 6 holds because $\sigma^{\prime}\left(p^{\prime}\right)=\sigma(p)$ and $\sigma(p) \models \varphi_{2}$.

- Condition 7 holds since $\sigma^{\prime}(j-1)=\sigma(j)$ and $\sigma(j) \models \varphi_{1}$ for all $j<p$.

- Condition 8 holds due to the fact that $W_{\sigma}(p) \leq k$ implies $W_{\sigma^{\prime}}\left(p^{\prime}\right) \leq$ $k-w_{i}$, because $W_{\sigma}(p)-W_{\sigma}^{\prime}\left(p^{\prime}\right)=w_{i}$.

As the $p^{\prime}$ constructed is strictly smaller than $p$, we have that $\rho\left(s_{i}, A \varphi_{1} U_{\leq k-w_{i}} \varphi_{2}\right)<\rho(s, \varphi)$. Thus, by induction it follows from $s_{i}=$ $A \varphi_{1} U_{\leq k-w_{i}} \varphi_{2}$ that $A_{\min }\left(s_{i}, A \varphi_{1} U_{\leq k-w_{i}} \varphi_{2}\right)=1$. As all configurations in a hyper-edge for $\langle s, \varphi\rangle$ are assigned the value 1 , it must hold that $A_{\min }(\langle s, \varphi\rangle)=1$.

(VII) For $\varphi=E X_{\leq k} \varphi$ we show that $A_{\min }\left(\left\langle s, E X_{\leq k} \varphi\right\rangle\right)=1$ if and only if $s \models E X_{\leq k} \varphi$ for all $s \in S$.

$\Rightarrow$ : Assume that $A_{\min }\left(\left\langle s, E X_{\leq k} \varphi\right\rangle\right)=1$, then it holds that $s \models E X_{\leq k} \varphi$. In Figure 2(g), the configuration $\left\langle s, E X_{\leq k} \varphi\right\rangle$ has a hyper-edge for every $s_{i} \in\left\{s_{i} \mid s \stackrel{w_{i}}{\rightarrow} s_{i}\right.$ and $\left.w_{i} \leq k\right\}$. Clearly, $A_{\min }\left(\left\langle s, E X_{\leq k} \varphi\right\rangle\right)=1$ if and only if $A_{\min }\left(\left\langle s_{i}, \varphi\right\rangle\right)=1$ is the case for any such $s_{i}$. By the induction hypothesis this is equivalent to $s_{i} \models \varphi$, which following the semantics implies that $s \models E X_{<k} \varphi$.

$\Leftarrow$ : Assume that $s \models E X_{\leq k} \varphi$, then it holds that $A_{\min }\left(\left\langle s, E X_{\leq k} \varphi\right\rangle\right)=1$. From the semantics, it must be the case that there exists an $s_{i}$, such that 
$s \stackrel{w_{i}}{\rightarrow} s_{i}$, with $w_{i} \leq k$, it holds that $s_{i}=\varphi$. By the induction hypothesis, this implies that $A_{\min }\left(\left\langle s_{i}, \varphi\right\rangle\right)=1$ for any such $s_{i}$. Since $A_{\min }$ is a pre fixed-point assignment, a hyper-edge in Figure 2(g) ensures that $A_{\min }\left(\left\langle s, E X_{\leq k} \varphi\right\rangle\right)=1$.

(VIII) For $\varphi=A X_{\leq k} \varphi$ we show that $A_{\min }\left(\left\langle s, A X_{\leq k} \varphi\right\rangle\right)=1$ if and only if $s \models A X_{\leq k} \varphi$ for all $s \in S$.

$\Rightarrow$ : Assume that $A_{\min }\left(\left\langle s, A X_{\leq k} \varphi\right\rangle\right)=1$, then it holds that $s=A X_{\leq k} \varphi$. In Figure 2(h), the configuration $\left\langle s, A X_{\leq k} \varphi\right\rangle$ has a single hyper-edge with a target set on the form $\left\{\left\langle s_{1}, \varphi\right\rangle, \ldots,\left\langle s_{n}, \varphi\right\rangle\right\}$, for every $s_{i}$, such that $s \stackrel{w_{i}}{\rightarrow} s_{i}$ and $w_{i} \leq k$. It is clear that $A_{\min }\left(\left\langle s, A X_{\leq k} \varphi\right\rangle\right)=1$ if and only if $A_{\min }\left(\left\langle s_{i}, \varphi\right\rangle\right)=1$ for all such $s_{i}$. Given the induction hypothesis, we have that $s_{i} \models \varphi$ for $1 \leq i \leq n$, which implies that $s \models A X_{\leq k} \varphi$.

$\Leftarrow$ : Assume that $s=A X_{\leq k} \varphi$, then it holds that $A_{\min }\left(\left\langle s, A X_{\leq k} \varphi\right\rangle\right)=1$. By the semantics it must be that case that $s_{i} \models \varphi$, for all $s_{i}$ such that $s \stackrel{w_{i}}{\rightarrow} s_{i}$, where $w_{i} \leq k$. By the induction hypothesis this implies that $A_{\min }\left(\left\langle s_{i}, \varphi\right\rangle\right)=1$ for all such $s_{i}$. As $A_{\min }$ is a pre fixed-point assignment, the hyper-edge in Figure 2(h) ensures that $A_{\min }\left(\left\langle s, A X_{\leq k} \varphi\right\rangle\right)=1$.

\section{A.2 Proofs related to symbolic dependency graphs}

We start with a technical lemma. In what follows, we shall use the notation $\mathcal{F}^{i}$ standing for $F^{i}\left(A_{0}\right)$.

Lemma 2. Let $G=(V, H, \emptyset)$ be an $S D G$ without cover-edges and $c_{i}$ denote a configuration which assignment changed to the smallest value in the $i$ 'th iteration of the functor, formally written as follows.

$$
c_{i}=\underset{v \in\left\{v \in V \mid \mathcal{F}^{i-1}(v)>F^{i}(v)\right\}}{\arg \min } \mathcal{F}^{i}(v)
$$

It holds that $\mathcal{F}^{i}\left(c_{i}\right)=A_{\text {min }}\left(c_{i}\right)$.

Proof. To prove that $A_{\min }\left(c_{i}\right)=\mathcal{F}^{i}\left(c_{i}\right)$, we show that Equation (16) holds. It then trivially follows that $\mathcal{F}^{i}\left(c_{i}\right)$ is the minimum pre fixed-point assignment of $c_{i}$, because no future smallest assignment in any iteration $j>i$ becomes less than $\mathcal{F}^{i}\left(c_{i}\right)$.

To show that Equation (16) holds, we observe that when the assignment of configuration $c_{i+1}$ is changed to the smallest value in the $i+1$ 'th iteration, then its assignment must have become smaller in iteration $i+1$, written as Equation (9).

$$
\begin{aligned}
\mathcal{F}^{i}\left(c_{i+1}\right) & >\mathcal{F}^{i+1}\left(c_{i+1}\right) \\
\mathcal{F}^{i+1}\left(c_{i+1}\right) & =\max \left\{w^{\prime}+\mathcal{F}^{i}\left(u^{\prime}\right) \mid\left(w^{\prime}, u^{\prime}\right) \in T\right\} \\
\mathcal{F}^{i-1}(u) & >\mathcal{F}^{i}(u) \\
\mathcal{F}^{i}(u) & \geq \mathcal{F}^{i}\left(c_{i}\right)
\end{aligned}
$$


This implies that there exists a hyper-edge $\left(c_{i+1}, T\right) \in H$ such that Equation (10) holds. Because the value $\mathcal{F}^{i+1}\left(c_{i+1}\right)$ was not reached in the $i$ 'th iteration, there must be a hyper-edge branch $(w, u) \in T$ such that the assignment of configuration $u$ changed from the $i-1$ 'th to the $i^{\prime}$ th iteration, which yields Equation (11).

We know that the smallest assignment changed from the $i-1$ 'th to the $i$ 'th iteration is $F^{i}\left(c_{i}\right)$. Hence, we get Equation (12), because no other assignment made in the $i$ 'th iteration is smaller than $F^{i}\left(c_{i}\right)$.

$$
\begin{aligned}
\max \left\{w^{\prime}+\mathcal{F}^{i}\left(u^{\prime}\right) \mid\left(w^{\prime}, u^{\prime}\right) \in T\right\} & \geq w+\mathcal{F}^{i}(u) \\
\mathcal{F}^{i+1}\left(c_{i+1}\right) & \geq w+\mathcal{F}^{i}(u) \\
\mathcal{F}^{i+1}\left(c_{i+1}\right) & \geq w+\mathcal{F}^{i}\left(c_{i}\right) \\
\mathcal{F}^{i+1}\left(c_{i+1}\right) & \geq \mathcal{F}^{i}\left(c_{i}\right)
\end{aligned}
$$

As the hyper-edge branch $(w, u)$ for which the value of $u$ changed is in $T$, we observe that $w+\mathcal{F}^{i}(u)$ must be less than equal to the right hand side of Equation (10) giving us Equation (13). Substituting this back into Equation (10) and we get Equation (14). We now recall the lower-bound on $\mathcal{F}^{i}(u)$ from Equation (12) in order to write Equation (15). Thus, we get Equation (16) as w must be non-negative.

\section{Proof of Theorem 3}

Computing the minimum pre fixed-point assignment of $G=(V, H, C)$ by repeated application of the functor $F$ takes $O(|V| \cdot|C| \cdot(|H|+|C|))$ time.

Proof. Let us first realize that a single iteration of $F$ takes $O(|H|+|C|)$ as we go through all the edges and and for each such edge update the value of the source configuration. Note that from the construction we have that there are always more configurations than cover-edges. After we establish that the algorithm terminates after no more than $|V| \cdot|C|$ iterations, the claim is proved.

If we consider a symbolic dependency graph without cover-edges $G=(V, H, \emptyset)$, we have that the minimum pre fixed-point assignment is reached within $|V|$ iterations. This follows from Lemma 2 that states that after each iteration, there is at least one configuration that reaches its minimum pre fixed point assignment.

Assume now that the symbolic dependency graph contains cover-edges. It is clear that once the value of a source configuration for a cover-edge is updated, it takes the value 0 and cannot be improved any more. Hence, after at most $|V|$ iterations at least one cover-edge sets the value of its source configuration to 0 and then we need to perform at most $|V|$ iterations before the same happens for another cover-edge, etc. Hence the total number of iterations is $O(|V| \cdot|C|)$ as required for establishing the claim of the theorem.

\section{A.3 Correctness of local algorithm on SDG}

\section{Proof of Lemma 1}

The while-loop in Algorithm 2 satisfies the following loop-invariants (for all configurations $v \in V$ ): 
1) If $A(v) \neq \perp$ then $A(v) \geq A_{\min }(v)$.

2) If $A(v) \neq \perp$ and $e=(v, T) \in H$, then either

a) $e \in W$,

b) $e \in D(u)$ and $A(v) \leq x$ for some $(w, u) \in T$ s.t. $x=A(u)+w$, where $x \geq A\left(u^{\prime}\right)+w^{\prime}$ for all $\left(w^{\prime}, u^{\prime}\right) \in T$, or

c) $A(v)=0$.

3) If $A(v) \neq \perp$ and $e=(v, k, u) \in C$, then either

a) $e \in W$,

b) $e \in D(u)$ and $A(u)>k$, or

c) $A(v)=0$.

Proof. We prove the invariants with the inductive argument that if the invariant holds at the beginning of the every iteration of the while-loop, it also holds at the end of every iteration.

Invariant (1): Initially, we have that $A(v)=\perp$, for all $v \in V \backslash\left\{v_{0}\right\}$, and $A\left(v_{0}\right)=\infty$ for the initial configuration $v_{0}$. Hence, the invariant holds trivially the first time the while-loop is entered.

We observe that the assignment $A$ is only updated in lines 10, 14, 20 and 22 of Algorithm 2. From this, there are three different cases to consider regarding the updated value of $A$.

In lines 10 and $20, A(v)$ is assigned the value $\infty$. Because $A(v)=\infty \geq$ $A_{\min }(v)$, it is clear that the invariant holds.

In line $14, A(v)$ is assigned the value $\max \{A(u)+w \mid(w, u) \in T\}$ for a hyper-edge $(v, T)$, if the value of this expression is strictly smaller than the current value of the assignment of $v$. This corresponds to the "otherwise" case of the function in Equation 1, hence the invariant holds.

In line $22, A(v)$ is assigned the value 0 , if there exists a cover-edge $(v, k, u)$ where $A(u) \leq k$, which corresponds to the first case of the functor in Equation 1. Thus, we have shown that Invariant (1) holds.

Invariants (2) and (3): The two invariants hold initially, because $A(v)=\perp$ for all $v \in V \backslash\left\{v_{0}\right\}$ and for the initial configuration $v_{0}$, we have that $W=$ $\operatorname{succ}\left(v_{0}\right)$. So, every hyper-/cover-edge with the source configuration $v_{0}$ is in $W$, which gives rise to cases (2a) and (3a).

We observe that, whenever a hyper-/cover-edge $e$ is removed from $W$, it is added to the dependency set $D(u)$ of a target configuration $u$ in $e$, unless it is the case that $A(v)$ has the value 0 . With this observation, and the fact that when we explore a new configuration $u$ by setting $A(u)=\infty$, we always add $\operatorname{succ}(u)$ to $W$. It is easy to see that Invariants 2 and 3 hold.

Theorem 7 (Algorithm 2 Termination). Algorithm 2 terminates.

Proof. The while-loop in Algorithm 2 finishes when the queue $W$ is empty ( $W=$ $\emptyset)$, resulting in the termination of Algorithm 2. To prove that this eventually occurs, we observe that whenever cover-/hyper-edges are added to $W$, then in the same iteration, there is a configuration $v$ such that the value of $A(v)$ decreases or $A(v)$ changes from $\perp$ to $\infty$. Moreover, we notice that $A(v)$ is always nonincreasing and once the value of $A(v)$ changes from $\perp$, it is never assigned the 
value $\perp$ again. Due to the fact that it is always the case that $A(v) \geq 0$, then it follows that in Algorithm 2, the cover-/hyper-edges are only added to $W$ a finite number of times. Thus, Algorithm 2 must terminate.

Theorem 8 (Algorithm 2 Correctness). Upon termination of Algorithm 2 on the input a symbolic dependency graph $G=(V, H, C)$, it holds that $A(v) \neq \perp$ implies $A(v)=A_{\min }(v)$ for all $v \in V$.

Proof. We prove correctness of Algorithm 2 by examining the cases of Lemma 1.

From Invariant (1) of Lemma 1, we have that for all $v \in V$, where $A(v) \neq \perp$, it holds that $A(v) \geq A_{\min }(v)$, leaving us to show that $A(v)$ is also a pre fixedpoint assignment.

To prove that $A(v)$ is pre fixed-point assignment for all $v \in V$ where $A(v) \neq$ $\perp$, we must show that $A(v)=F(A)(v)$. From the definition of the functor (Equation 1) we see that the two following cases must be considered.

i) If there exists $(v, k, u) \in C$ and $A(u) \leq k$, then $A(v)=0$.

ii) For any $(v, T) \in H$ and $x=\max \left\{A\left(u^{\prime}\right)+w^{\prime} \mid\left(w^{\prime}, u^{\prime}\right) \in T\right\}$, then $A(v) \leq x$.

First we consider case (i). We prove by contradiction that $A(v)=0$. Assume that $A(v)>0$. Considering invariant 3 , we observe that the algorithm has terminated, thus, $W=\emptyset$ and case 3 a cannot hold. This means that either case $3 \mathrm{~b}$ or case $3 \mathrm{c}$ must hold. Since $A(v) \neq 0$, we know that case $3 \mathrm{c}$ does not hold, leaving us to conclude that case $3 \mathrm{~b}$ holds. By case $3 \mathrm{~b}$, we have $A(u)>k$, which contradicts $A(u) \leq k$. Thus, we must have $A(v)=0$, proving (i).

For case (ii). We prove by contradiction that $A(v) \leq x$. Assume that $A(v)>x$. Considering Invariant 2, we observe as before that the algorithm has terminated, thus, $W=\emptyset$ and case 2 a cannot hold. Hence, either case $2 \mathrm{~b}$ or case $2 \mathrm{c}$ must hold. Because $x \geq 0$ and we assumed $A(v)>x$, it must be the case that $A(v) \neq 0$, so we know that case $2 \mathrm{c}$ cannot hold. This leaves us with case $2 \mathrm{~b}$, which by Lemma 1 must hold.

By case $2 \mathrm{~b}$, we have that there exists a hyper-edge branch $(w, u) \in T$, s.t. $A(v) \leq x^{\prime}$, where $x^{\prime}=A(u)+w$ and $x^{\prime} \geq A\left(u^{\prime}\right)+w^{\prime}$ for all $\left(w^{\prime}, u^{\prime}\right) \in T$. As both $x$ and $x^{\prime}$ are the maximum value of the set $\left\{A\left(u^{\prime}\right)+w^{\prime} \mid\left(w^{\prime}, u^{\prime}\right) \in T\right\}$, it must be the case that $x=x^{\prime}$. Thus, $A(v) \leq x^{\prime}$ contradicts our assumption that $A(v)>x$. Therefore, it must be the case that $A(v) \leq x$.

Consequently, we conclude that upon termination of Algorithm 2, it holds that for all $v \in V$, where $A(v) \neq \perp$, the assignment $A(v)$ is the minimum pre fixed-point assignment of $v$.

Corollary 1. Given a symbolic dependency graph $G=(V, H, C)$ and an initial configuration $v_{0} \in V$, Algorithm 2 computes the minimum pre fixed-point assignment of $v_{0}$ in $G$.

Proof. We only assign $\perp$ in line 1 and since $A\left(v_{0}\right)$ is assigned $\infty$ initially, we cannot have $A\left(v_{0}\right)=\perp$ upon when finishing the while-loop. Thus, in line 27 of Algorithm 2 we have $A\left(v_{0}\right)=A_{\min }\left(v_{0}\right)$ by Theorem 8. Consequently, Algorithm 2 returns the minimum pre fixed-point assignment of $v_{0}, A_{\min }\left(v_{0}\right)$. 


\section{A.4 Correctness of Encoding of WCTL Model Checking into SDG}

\section{Proof of Theorem 5}

Let $\mathcal{K}=(S, \mathcal{A P}, L, \rightarrow)$ be a WKS, $s \in S$ a state, $\varphi$ a WCTL formula. Let $G$ be the constructed symbolic dependency graph rooted with $\langle s, \varphi\rangle$. Then $s=\varphi$ if and only if $A_{\min }(\langle s, \varphi\rangle)=0$.

Proof. We prove Theorem 5 by observing that there is two kinds of configurations in the symbolic dependency graph rooted with $\langle s, \varphi\rangle$. We have that configurations on the form $\left\langle s, E \varphi_{1} U_{\leq ?} \varphi_{2}\right\rangle$ or $\left\langle s, A \varphi_{1} U_{\leq ?} \varphi_{2}\right\rangle$ may have non-zero hyper-edge weights. We shall refer to these configurations as symbolic configurations, and all other configurations as concrete configurations.

Notice that the bound for symbolic configurations is "?", while $\left\langle s, E \varphi_{1} U_{\leq k} \varphi_{2}\right\rangle$ is a concrete configuration. With the introduction of concrete and symbolic configurations, we now present two invariants for the symbolic encoding.

i) Concrete configurations $\langle s, \varphi\rangle$ can only obtain the values 0 or $\infty$, where $A_{\min }(\langle s, \varphi\rangle)=0$ if and only if $s=\varphi$.

ii) For a symbolic configuration $v=\left\langle s, E \varphi_{1} U_{\leq ?} \varphi_{2}\right\rangle$ it holds that $A_{\min }(v)=k$ if and only if $s=E \varphi_{1} U_{\leq k^{\prime}} \varphi_{2}$ for any $k^{\prime} \geq k$.

(A similiar invariant applies to configurations for the universal until-formula).

It is easy to see that Theorem 5 follows trivially from Invariant (i). Thus, we need only show that these invariants hold by structural induction on $\varphi$.

(I) For $\varphi=$ true we show that Invariant (i) holds for all configurations $\langle s$, true $\rangle$. Because $s \models$ true always holds we need only show that $A_{\text {min }}(\langle s$, true $\rangle)=0$. In Figure 2(a) there is a hyper-edge from the configuration $\langle s$, true $\rangle$ to the empty target set. Hence, we have that $A(v)=0$ for any pre fixed-point assignment $A$ of $G$.

(II) For $\varphi=a$ we prove Invariant (i), i.e. $A_{\min }(\langle s, a\rangle)=0$ if and only if $s \models a$ for all $s \in S$. If $a \in L(s)$ we have $s \models a$ and by Figure 2(b), there is a hyper-edge from the configuration $\langle s, a\rangle$ to the empty target set. Like in the previous case this means that $A_{\min }(\langle s, a\rangle)=0$, which leaves us to consider the case when $a \notin L(s)$. In this case it is clear that $s \not \models a$ and by the side-condition in Figure 2(b), we can conclude that there is no hyper-edge from the configuration $\langle s, a\rangle$ when $a \notin L(s)$. Thus, we have $A_{\min }(\langle s, a\rangle)=\infty$ since $A_{\min }$ is the minimum pre fixed-point assignment.

(III) For $\varphi=\varphi_{1} \wedge \varphi_{2}$ we show that Invariant (i) holds. First we show that $A_{\min }\left(\left\langle s, \varphi_{1} \wedge \varphi_{2}\right\rangle\right)$ is either $\infty$ or 0 , and $A_{\min }\left(\left\langle s, \varphi_{1} \wedge \varphi_{2}\right\rangle\right)=0$ if and only if $s \models \varphi_{1} \wedge \varphi_{2}$. Since sub-configurations $\left\langle s, \varphi_{1}\right\rangle$ and $\left\langle s, \varphi_{1}\right\rangle$ are concrete (Figure 2(c)) it follows by structural induction that their assignments only evaluate to either 0 or $\infty$. Furthermore, we have $A_{\min }\left(\left\langle s, \varphi_{1}\right\rangle\right)=0$ and $A_{\text {min }}\left(\left\langle s, \varphi_{2}\right\rangle\right)=0$, if and only if $s \models \varphi_{1}$ and $s \models \varphi_{2}$, which following the semantics implies $s \models \varphi_{1} \wedge \varphi_{2}$.

(IV) For $\varphi=\varphi_{1} \vee \varphi_{2}$ Invariant (i) can be shown with arguments similar to those used previously for conjunction. 
(V) For $\varphi=E \varphi_{1} U_{\leq k} \varphi_{2}$ we show Invariant (i), i.e. $A_{\min }\left(\left\langle s, E \varphi_{1} U_{\leq k} \varphi_{2}\right\rangle\right)=$ 0 if and only if $s=E \varphi_{1} U_{\leq k} \varphi_{2}$ for all $s \in S$. From Figure 6(a) we see that any configuration $\left\langle s, E \varphi_{1} U_{\leq k} \varphi_{2}\right\rangle$ has a single cover-edge with the covercondition $k$ leading to the symbolic configuration $v=\left\langle s, E \varphi_{1} U_{\leq ?} \varphi_{2}\right\rangle$. By structural induction we have from Invariant (ii) that $A_{\min }(v) \leq k$ if and only if $s \models E \varphi_{1} U_{\leq k} \varphi_{2}$. Thus, we have shown Invariant (i), as cover-edges can only assign the value 0 .

(VI) For $\varphi=E \varphi_{1} U_{\leq ?} \varphi_{2}$ we show Invariant (ii), i.e. that $A_{\min }\left(\left\langle s, E \varphi_{1} U_{\leq ?} \varphi_{2}\right\rangle\right)=$ $k$ if and only if $s \models E \varphi_{1} U_{\leq k^{\prime}} \varphi_{2}$ for any $k^{\prime} \geq k$.

Recall the semantics for the satisfaction of the formula $E \varphi_{1} U_{\leq k} \varphi_{2}$, requires that for some $k^{\prime} \leq k$, there exists a run $\sigma$ and a position $p \geq 0$ satisfying the following conditions.

$$
\begin{aligned}
\sigma(p) & \models \varphi_{2} \\
\sigma(j) & \models \varphi_{1}, \text { for all } j<p \\
W_{\sigma}(p) & \leq k^{\prime}
\end{aligned}
$$

$\Rightarrow$ : Assume that $A_{\min }\left(\left\langle s, E \varphi_{1} U_{\leq ?} \varphi_{2}\right\rangle\right)=k$, we now show that this implies the existence of a run $\sigma$ and position $p$ satisfying conditions 17, 18 and 19 for $k^{\prime} \leq k$. By the semantics this obviously implies $s=E \varphi_{1} U_{\leq k^{\prime}} \varphi_{2}$ for any $k^{\prime} \geq k$.

We denote the iteration in which a configuration $v$ was first assigned the value $k$, as $Z_{k}(v)$. Formally we write the auxiliary function $Z_{k}$ as follows.

$$
Z_{k}(v)= \begin{cases}i & \text { if } F^{i}(v) \leq k \text { and } F^{i-1}(v)>k \\ \infty & \text { otherwise }\end{cases}
$$

For any configuration $v$ it holds that $Z_{k}(v)<\infty$ if and only if $A_{\min }(v) \leq k$, as a fixed-point must be reached in a finite number of iterations. Considering $Z_{k}(v)$ for a configuration $v=\left\langle s, E \varphi_{1} U_{\leq ?} \varphi_{2}\right\rangle$, where $A_{\min }(v) \leq k$, we see that in iteration $Z_{k}(v)-1$, the assignment of some configuration in the target-set for a hyper-edge to $v$ must have been changed to $k$. From Figure 2(e) we see that there are two kinds of hyper-edges, leading us to conclude that at least one of the following two cases must hold.

A) $Z_{k}\left(\left\langle s, \varphi_{2}\right\rangle\right)=Z_{k}(v)-1$, or

B) $\max \left\{Z_{k}\left(\left\langle s, \varphi_{1}\right\rangle\right), Z_{k-w}\left(\left\langle s^{\prime}, E \varphi_{1} U_{\leq ?} \varphi_{2}\right\rangle\right)\right\}=Z_{k}(v)-1$, for some $s^{\prime}$, s.t. $s \stackrel{w}{\rightarrow} s^{\prime}$.

We now show that $A_{\min }\left(\left\langle s, E \varphi_{1} U_{\leq ?} \varphi_{2}\right\rangle\right)=k$ implies the existence of a run $\sigma$ and a position $p$ satisifying conditions 17,18 and 19 for $k^{\prime} \leq k$, by induction on $Z_{k}\left(\left\langle s, E \varphi_{1} U_{\leq ?} \varphi_{2}\right\rangle\right)$.

First we observe that $Z_{k}\left(\left\langle s, E \varphi_{1} U_{\leq ?} \varphi_{2}\right\rangle\right)$ is always greater than 1 , as only configurations $v$ having trivial hyper-edges $(v, \emptyset)$ are assigned 0 in the first iteration of $F$.

Base Case $\left(Z_{k}\left(\left\langle s, E \varphi_{1} U_{\leq ?} \varphi_{2}\right\rangle\right)=2\right)$ : In this case we know that case (A) must hold, seeing that no configuration $u=\left\langle s^{\prime}, E \varphi_{1} U_{\leq}\right.$? $\left.\varphi_{2}\right\rangle$ can have $Z_{k-w}(u)=1$. From case (A), we have that $Z_{k}\left(\left\langle s, \varphi_{2}\right\rangle\right)=1$ and as this 
is a concrete configuration, it holds that $A_{\min }\left(\left\langle s, \varphi_{2}\right\rangle\right)=0$ by Invariant i. From here it also follows that $A_{\min }\left(\left\langle s, \varphi_{2}\right\rangle\right)=0$ implies $s=\varphi_{2}$. Thus, any run $\sigma=s \ldots$ and position $p=0$ satisfy conditions 17,18 and 19 for $k^{\prime}=0$, hence, it also holds for $k \geq k^{\prime}$.

Inductive Step $\left(Z_{k}\left(\left\langle s, E \varphi_{1} U_{\leq ?} \varphi_{2}\right\rangle\right)>2\right)$ : Again, we consider cases (A) and (B). If case $(A)$ holds we can construct a run $\sigma=s \ldots$ and position $p=0$ as before. If (B) is the case, we have that $Z_{k}\left(\left\langle s, \varphi_{1}\right\rangle\right) \leq \infty$ which implies $A_{\min }\left(\left\langle s, \varphi_{1}\right\rangle\right)=0$ as $\left\langle s, \varphi_{1}\right\rangle$ is a concrete configuration. Futhermore, it follows from Invariant (ii) by structural induction that $s \models \varphi_{1}$.

Because $Z_{k-w}\left(\left\langle s^{\prime}, E \varphi_{1} U_{\leq ?} \varphi_{2}\right\rangle\right)<Z_{k}\left(\left\langle s, E \varphi_{1} U_{\leq ?} \varphi_{2}\right\rangle\right)$ it follows by induction that there is a run $\sigma=s^{\prime} \ldots$ and a position $p$ that satisfy conditions 17, 18 and 19 for $k^{\prime} \leq k-w$. Considering the extension $\sigma^{\prime}=$ $s \stackrel{w}{\rightarrow} s^{\prime} \ldots$ of $\sigma$ and position $p^{\prime}=p+1$, we observe that $\sigma^{\prime}$ and $p^{\prime}$ also satisfy the conditions for $k^{\prime} \leq k$.

- Condition 17 holds because $\sigma^{\prime}\left(p^{\prime}\right)=\sigma(p)$ and $\sigma(p) \mid=\varphi_{2}$.

- Condition 18 holds since $\sigma(0)=s, s \models \varphi_{1}$ and for all $j<p$ we have $\sigma^{\prime}(j+1)=\sigma(j)$ and $\sigma(j) \models \varphi_{1}$.

- Condition 19 holds due to the fact that $W_{\sigma}(p) \leq k-w$ implies $W_{\sigma^{\prime}}\left(p^{\prime}\right) \leq k$, because $W_{\sigma^{\prime}}\left(p^{\prime}\right)-W_{\sigma}(p)=w$.

$\Leftarrow$ : Assume that $s \models E \varphi_{1} U_{\leq k} \varphi_{2}$, we now show that this implies $A_{\min }\left(\left\langle s, E \varphi_{1} U_{\leq ?} \varphi_{2}\right\rangle\right) \leq k$. From the semantics it follows that there is a run $\sigma$ and position $p$ satisfying conditions 17, 18 and 19 for $k^{\prime} \leq k$ Let $s=s_{0}$, then we can write $\sigma$ as follows.

$$
\sigma=s_{0} \stackrel{w_{1}}{\rightarrow} s_{1} \ldots s_{p-1} \stackrel{w_{p}}{\rightarrow} s_{p} \ldots
$$

We show that $A_{\min }\left(\left\langle s_{i}, E \varphi_{1} U_{\leq ?} \varphi_{2}\right\rangle\right) \leq k-W_{\sigma}(i)$ by induction on $i$ starting from $p$.

Base Case $(i=p)$ : By condition 17 of the semantics, $s_{p} \models \varphi_{2}$, which by structural induction on $\varphi$ implies $A_{\min }\left(\left\langle s_{p}, \varphi_{2}\right\rangle\right)=0$ because $\left\langle s_{p}, \varphi_{2}\right\rangle$ is a concrete configuration. In Figure 6(c), we observe that there is a hyperedge from $\left\langle s_{p}, E \varphi_{1} U_{\leq ?} \varphi_{2}\right\rangle$ to $\left\langle s_{p}, \varphi_{2}\right\rangle$, thus, $A_{\min }\left(\left\langle s_{p}, \varphi_{2}\right\rangle\right)=0$ implies $A_{\text {min }}\left(\left\langle s_{p}, E \varphi_{1} U_{\leq 0} \varphi_{2}\right\rangle\right)=0$, which proves our base case.

Inductive Step $(i<p)$ : By condition 18 of the semantics, $s_{i}=\varphi_{1}$, which by structural induction on $\varphi$ implies $A_{\min }\left(\left\langle s_{i}, \varphi_{1}\right\rangle\right)=0$. By induction on $i$, we know that $A_{\min }\left(\left\langle s_{i+1}, E \varphi_{1} U_{\leq ?} \varphi_{2}\right\rangle\right) \leq k-W_{\sigma}(i+1)$ holds.

In Figure 6(c), we observe that there is a hyper-edge $e$ from $\left\langle s_{i}, E \varphi_{1} U_{\leq \text {? }} \varphi_{2}\right\rangle$ to the target-set $\left\langle s_{i}, \varphi_{1}\right\rangle$ and $\left\langle s_{i+1}, E \varphi_{1} U_{\leq ?} \varphi_{2}\right\rangle$. We also notice that $e$ has the transition weight between $s_{i}$ and $s_{i+1}, w_{i+1}$, on the hyper-edge branch to $\left\langle s_{i+1}, E \varphi_{1} U_{\leq ?} \varphi_{2}\right\rangle$. Thus, from the semantics of hyper-edges it follows that $A_{\min }\left(\left\langle s_{i}, E \varphi_{1} U_{\leq ?} \varphi_{2}\right\rangle\right) \leq k-W_{\sigma}(i+1)+w_{i+1}$. But as $W_{\sigma}(i)+w_{i+1}=W_{\sigma}(i)$ we have that $A_{\min }\left(\left\langle s_{i}, E \varphi_{1} U_{\leq ?} \varphi_{2}\right\rangle\right) \leq k-W_{\sigma}(i)$, which proves our inductive case.

(VII) For $\varphi=A \varphi_{1} U_{\leq k} \varphi_{2}$ we have that $A_{\min }\left(\left\langle s, E \varphi_{1} U_{\leq k} \varphi_{2}\right\rangle\right)=0$ if and only if $s \models A \varphi_{1} U_{\leq k} \varphi_{2}$ for all $s \in S$. The proof strategy here is similar to the previously shown case for $\varphi=E \varphi_{1} U_{\leq k} \varphi_{2}$. 
(VIII) For $\varphi=A \varphi_{1} U_{\leq ?} \varphi_{2}$ it can be shown that $A_{\min }\left(\left\langle s, E \varphi_{1} U_{\leq ?} \varphi_{2}\right\rangle\right)=k$ if and only if $s \models A \varphi_{1} U_{\leq k^{\prime}} \varphi_{2}$ for all $k^{\prime} \geq k$. The proof strategy is an adaptation of the approach for ordinary dependency graphs. In particular it is similar to the proof strategy applied for $E \varphi_{1} U_{\leq ?} \varphi_{2}$, which was adapted from the proof for ordinary dependency graphs.

(IX) For $\varphi=E X_{\leq k} \varphi$ we observe in Figure 2(g) that all successor configurations are concrete. It is straightforward to adapt the proof strategy used for ordinary dependency graphs for this case.

(X) For $\varphi=A X_{\leq k} \varphi$, shown in Figure 2(h), all successor configurations are again conrete. Once again, it is easy to adapt the proof strategy for this case.

\section{A.5 NP-Hardness of WCTL with Interval Bounds}

We show NP-hardness of satisfiability of until-formulas with interval bounds by reduction from the subset-sum problem.

Definition 3 (Subset-Sum Problem). Let $W=\left\{w_{1}, \ldots, w_{n}\right\}$ be a set of integers and $T$ be a target integer, is there a vector $\boldsymbol{x} \in \mathbb{N}_{0}^{n}$, s.t.

$$
\sum_{i=1}^{n} w_{i} \cdot x_{i}=T ?
$$

The variation of the subset-sum problem presented above is known to be NP-complete [17, Chap. 5].

Given an instance $W, T$ of the subset-sum problem, we construct a WKS $\mathcal{K}$ as shown in Figure 8. It is easy to check that the formula $s \models E$ true $U_{[T, T]}$ true is satisfied if and only if $W, T$ is a positive instance of the subset-sum problem.

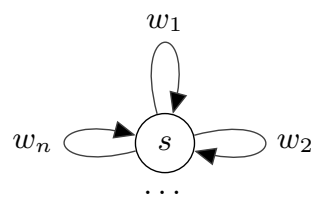

Fig. 8. Subset-sum construction 